\title{
A Godunov-Type Solver for the Numerical Approx- imation of Gravitational Flows
}

\author{
J. Vides ${ }^{1, *}$, B. Braconnier ${ }^{2}$, E. Audit ${ }^{3}$, C. Berthon ${ }^{4}$, and B. Nkonga ${ }^{5,6}$ \\ ${ }^{1}$ Inria, Maison de la Simulation, USR 3441, Gif-sur-Yvette, France. \\ 2 IFP Energies Nouvelles, 1-4 avenue de Bois-Préau, 92852 Rueil-Malmaison, France. \\ ${ }^{3}$ CEA, Maison de la Simulation, USR 3441, Gif-sur-Yvette, France. \\ 4 Univ. of Nantes, Lab. J. Leray, UMR CNRS 6629, Nantes, France. \\ ${ }^{5}$ Univ. of Nice-Sophia Antipolis, Lab. J.A. Dieudonné, UMR CNRS 7351, Nice,France. \\ ${ }^{6}$ Inria Sophia Antipolis, BP. 93, F-06902 Sophia Antipolis Cedex, France.
}

\begin{abstract}
We present a new numerical method to approximate the solutions of an Euler-Poisson model, which is inherent to astrophysical flows where gravity plays an important role. We propose a discretization of gravity which ensures adequate coupling of the Poisson and Euler equations, paying particular attention to the gravity source term involved in the latter equations. In order to approximate this source term, its discretization is introduced into the approximate Riemann solver used for the Euler equations. A relaxation scheme is involved and its robustness is established. The method has been implemented in the software HERACLES [29] and several numerical experiments involving gravitational flows for astrophysics highlight the scheme.
\end{abstract}

Key words: Euler-Poisson equations, approximate Riemann solver, relaxation scheme, source terms, gravitational effects.

\section{Introduction}

The present paper is devoted to the numerical approximation of the Euler equations when gravitational effects are taken into account. The associated solutions are governed by an Euler-Poisson model, given by the following system of partial differential equations (PDEs):

$$
\left\{\begin{array}{l}
\partial_{t} \rho+\nabla \cdot(\rho \mathbf{u})=0, \\
\partial_{t}(\rho \mathbf{u})+\nabla \cdot(\rho \mathbf{u} \otimes \mathbf{u}+p)=-\rho \nabla \phi, \\
\partial_{t}(\rho E)+\nabla \cdot((\rho E+p) \mathbf{u})=-\rho \mathbf{u} \cdot \nabla \phi, \\
\Delta \phi=4 \pi G \rho,
\end{array}\right.
$$

*Corresponding author. Email addresses: jeaniffer-lissette.vides_higueros@inria.fr (J. Vides), benjamin.braconnier@ifpenergiesnouvelles.fr (B. Braconnier), edouard.audit@cea.fr (E. Audit), christophe.berthon@math.univ-nantes.fr (C. Berthon), boniface.nkonga@unice.fr (B. Nkonga) 
where $\rho>0$ is the density, $\mathbf{u} \in \mathbb{R}^{d}$ the velocity, and $E$ the specific energy. The integer $d$ refers to the space dimension. The thermodynamic pressure $p$ is assumed to be governed by an equation of state

$$
p:=p(\rho, \epsilon),
$$

where $\epsilon=E-|\mathbf{u}|^{2} / 2$ represents the specific internal energy. As usual (see $\left.[28,49]\right)$, the pressure law is assumed to satisfy

$$
\partial_{\rho} p(\rho, \epsilon)+\frac{p(\rho, \epsilon)}{\rho^{2}} \partial_{\epsilon} p(\rho, \epsilon)>0 .
$$

Here, $G \approx 6.67 \times 10^{-11} \mathrm{~m}^{3} \mathrm{~kg}^{-1} \mathrm{~s}^{-2}$ is the gravitational constant. The gravitational potential $\phi$ is always a smooth function since it is the solution of the Laplace equation. For the sake of notation simplicity, the system is rewritten in compact form as

$$
\left\{\begin{array}{l}
\partial_{t} \mathbf{W}+\nabla \cdot \mathbf{F}(\mathbf{W})+\mathbf{B}(\mathbf{W}) \nabla \phi=0, \\
\Delta \phi=4 \pi G \rho,
\end{array}\right.
$$

with

$$
\begin{aligned}
\mathbf{W} & =(\rho, \rho \mathbf{u}, \rho E)^{T}, \\
\mathbf{F}(\mathbf{W}) & =(\rho \mathbf{u}, \rho \mathbf{u} \otimes \mathbf{u}+p,(\rho E+p) \mathbf{u})^{T}, \\
\mathbf{B}(\mathbf{W}) & =\rho\left(\begin{array}{c}
0_{d}^{T} \\
\mathcal{I}_{d} \\
\mathbf{u}^{T}
\end{array}\right),
\end{aligned}
$$

where $\mathbf{0}_{d}=(0, \ldots, 0)^{T}$ is the null vector in $\mathbb{R}^{d}$ and $\mathcal{I}_{d}$ is an identity matrix of dimension $d$. As usual, $\mathbf{W}: \mathbb{R}^{d} \times \mathbb{R}^{+} \rightarrow \Omega_{d}$ is the state vector and $\mathbf{F}: \Omega_{d} \rightarrow \mathbb{R}^{2+d}$ is the flux function. Here, $\mathbf{B}: \Omega_{d} \rightarrow \mathbb{R}^{2+d} \times \mathbb{R}^{d}$ represents the gravitational contribution when multiplied by $\nabla \phi$. The convex set $\Omega_{d}$ of the admissible state vectors is defined by

$$
\Omega_{d}=\left\{\mathbf{W} \in \mathbb{R}^{2+d} ; \rho>0, \mathbf{u} \in \mathbb{R}^{d}, \epsilon=E-\frac{|\mathbf{u}|^{2}}{2}>0\right\}
$$

System (1.2) is completed with appropriate initial and boundary conditions that depend on the problem being considered, as will be seen in Section 5. Nevertheless, we note that in the field of astrophysics, it is common to find isolated boundary conditions for the potential $\phi$, i.e., the potential is then approximated by a multipolar development of the mass distribution at the boundary (see [33]). For the initial data, we define

$$
\mathbf{W}(\mathbf{x}, 0)=\mathbf{W}^{0}(\mathbf{x}), \quad \phi(\mathbf{x}, 0)=\phi^{0}(\mathbf{x}),
$$

where the functions $\mathbf{W}^{0}(\mathbf{x})$ and $\phi^{0}(\mathbf{x})$ are chosen according to the specific physical settings of the problem addressed. 
The Euler-Poisson model is certainly one of the most commonly used fluid models in plasma physics. However, simulations of plasma flows are not the purpose of the present work and will not be discussed in detail. We briefly wish to note that an abundant literature is devoted to the Euler-Poisson problem in plasmas (for instance, see [20-25, $39,40])$. The equations are used to describe ions and electrons flows, which are usually highly dynamic. A particular issue resides in the quasi-neutral limit (equality of the ion and electron densities) where the Poisson equation becomes singular. The derivation of efficient numerical schemes in this limit is a complex task. In fact, the space and time scales of the solution tend toward zero, leading to severe consistency and stability criteria. In order to overcome this difficulty, Degond et al. [20,21, 23, 24] (see also [22]) proposed a reformulation of the Poisson equation which is not singular in the quasi-neutral limit. The resulting numerical scheme resolves successfully all the plasma regimes, which is particularly attractive when different regimes are present in a single domain.

However, in the field of astrophysical flows and fluid dynamics, the Poisson equation never bears singularities. The main challenge consists in discretizing the gravitational effects governed by $\mathbf{B}(\mathbf{W}) \nabla \phi$. In fact, in the limit of a steady flow, the first three equations of system (1.1) give

$$
\begin{cases}\nabla \cdot(\rho \mathbf{u}) & =0, \\ \nabla \cdot(\rho \mathbf{u} \otimes \mathbf{u}+p) & =-\rho \nabla \phi, \\ \nabla \cdot((\rho E+p) \mathbf{u}) & =-\rho \mathbf{u} \cdot \nabla \phi .\end{cases}
$$

The main difficulty then resides in the design of a numerical scheme that preserves this asymptotic regime accurately. To address this issue, particular attention must be paid to the gravitational effects $\mathbf{B}(\mathbf{W}) \nabla \phi$.

We now focus on system (1.2). We use an operator splitting approach (Yanenko splitting, first-order, or Strang splitting, second-order accurate), and in the context of the firstorder decomposition, equation (1.2) is solved in two steps. First, we restrict our attention to the system

$$
\partial_{t} \mathbf{W}+\nabla \cdot \mathbf{F}(\mathbf{W})+\mathbf{B}(\mathbf{W}) \nabla \phi=0,
$$

where $\mathbf{W} \in \Omega_{d}$ is the unknown vector and the gravitational potential $\phi$ is an a priori given function. Equation (1.7) is in nonconservative form and the method used to solve it will be discussed later. Once the solution has been found, the second step consists in using the first component of $\mathbf{W}$, i.e., $\rho$, to solve the Poisson equation

$$
\Delta \phi=4 \pi G \rho,
$$

by means of a classical second-order finite difference approach. The operator splitting approach allows to decompose (1.2) into two subproblems and treat equations (1.7) and (1.8) individually, which are hyperbolic and elliptic, respectively.

Several numerical strategies can be adopted in view of solving (1.7). The first idea [37] is to decompose the solution into a hydrostatic steady part governed by system (1.6), and an acoustic dynamic part governed by the Euler equations without source terms. Classical Godunov-type based numerical schemes can be used to approximate the acoustic 
dynamic part. Unfortunately, this strategy tends to become much too complex in 3D or when a complete implicit formulation is required because of system (1.6) resolution.

The second idea is to construct a Godunov-type solver that takes into account the gravitational effects. A full conservative reformulation of (1.7) proposed in another context [3] (see also Chieze [18]) could be used. Indeed, the authors introduce the specific energy, denoted $E_{\phi}=E+\phi$, to rewrite (1.7) in the following conservative form:

$$
\left\{\begin{array}{lll}
\partial_{t}(\rho) & +\nabla \cdot(\rho \mathbf{u}) & =0 \\
\partial_{t}(\rho \mathbf{u}) & +\nabla \cdot\left(\rho \mathbf{u} \otimes \mathbf{u}+p+\frac{\nabla \phi \otimes \nabla \phi}{8 \pi G}\right) & =0 \\
\partial_{t}\left(\rho E_{\phi}+\frac{|\nabla \phi|^{2}}{8 \pi G}\right) & +\nabla \cdot\left(\rho E_{\phi} \mathbf{u}-\frac{\nabla \phi \partial_{t} \phi}{4 \pi G}\right) & =0
\end{array}\right.
$$

Despite the conservation form, the nonlinearities involved in the differential operators make this approach of little interest from a scientific computing point of view.

Another approach comes from the work of Greenberg et al. [30,31] where the potential form of the source term is used with some benefits to derive well-balanced schemes. Such a numerical procedure has been widely improved in the literature (for instance, see $[13,26,27,34,41]$ for the shallow-water equations, [10] in the framework of radiative transfer or [2] for extensions of the Kerr-Debye model), and constitutes a relevant alternative to discretize (1.7). However, the nonlinear Riemann problem involved by this approach is too sophisticated in the present framework to be directly considered. Hence, the extension of this technique to the present context is obtained by involving a relaxation scheme. This relaxation technique is adopted to introduce relevant linearizations and thus derive a Suliciu-type relaxation solver for the Euler-Poisson system. From now on, we note that the obtained scheme is not well-balanced in the sense of [30,31], but the steady states will be approximated with a better accuracy than with standard fractional step splitting approaches.

This paper is organized as follows. In the next section, we exhibit a relevant relaxation model to approximate the solutions of the system under consideration. One of the benefits of the proposed relaxation model is that it ensures a strong coupling between the Euler equations and the gravitational potential. The details of the Riemann solution associated with the homogeneous relaxation model are presented in Section 3. Section 4 is then devoted to the derivation of the relaxation scheme. Several numerical tests are presented and discussed in Section 5. Finally, concluding remarks are given in Section 6.

\section{1-D relaxation model}

We now consider the numerical approximation of system (1.7). For simplicity, the developments are proposed considering the 1-D model. The system can thus be written as

$$
\begin{cases}\partial_{t} \rho+\partial_{x}(\rho u) & \\ \partial_{t}(\rho u)+\partial_{x}\left(\rho u^{2}+p\right) & =0 \\ \partial_{t}(\rho E)+\partial_{x}((\rho E+p) u)+\rho u \partial_{x} \phi & =0\end{cases}
$$


where $\phi$ is an a priori given function, solution to the Poisson equation (1.8). With the definition of $\mathbf{W}, \mathbf{F}$ and $\mathbf{B}$ given by (1.3), we write the system under the form

$$
\partial_{t} \mathbf{W}+\partial_{x} \mathbf{F}(\mathbf{W})+\mathbf{B}(\mathbf{W}) \partial_{x} \phi=0,
$$

and the 1-D state vector belongs to the phase space $\Omega_{1}$ defined by (1.4).

The main difficulty lies with the source term, which requires an adequate approximation. We propose to derive a relaxation scheme, a classical numerical approach widely spread across the literature considering fluid flow simulations $[1,7-9,17,38]$. After the work of Jin-Xin [35] or Bouchut [13] (see also [4, 16, 19]), we propose to approximate the weak solutions of (2.1) by the weak solutions of a suitable first order PDE system with singular perturbations: the relaxation system. In order to make this strategy attractive, the relaxation system is generally designed to render the Riemann problem easily solvable. However, according to the work of Coquel-Perthame [19] (for instance, see also $[4,11])$, most of the nonlinearities of the initial system, called relaxation equilibrium system, must be preserved by the relaxation system to enforce accuracy of the resulting numerical scheme.

After the work of Suliciu $[43,44]$ (see also $[1,7-9,15,17,38]$ for several extensions), we first propose to relax the thermodynamic pressure $p$. In this sense, we suggest substituting $p$ by an approximation $\pi$ governed by a suitable evolution law supplemented by a relaxation source term. Such an evolution law can be deduced from the pressure equation. After usual computations (for instance, see [28]), the pressure equation is

$$
\partial_{t} p+u \partial_{x} p+\rho c^{2} \partial_{x} u=0,
$$

where the sound speed $c$ is defined as

$$
c^{2}=\partial_{\rho} p(\rho, \epsilon)+\frac{p(\rho, \epsilon)}{\rho^{2}} \partial_{\epsilon} p(\rho, \epsilon)>0 .
$$

Let us underline that relation (2.3) does not depend on the gravitational potential $\phi$. From (2.3), we suggest the following evolution equation to govern the additional variable $\pi$ :

$$
\partial_{t} \pi+u \partial_{x} \pi+\frac{a^{2}}{\rho} \partial_{x} u=\frac{1}{\delta}(p-\pi) .
$$

A sub-characteristic Whitham condition [50] will be considered later to fix the relaxation parameter $a$. The choice of $a$ will be done to satisfy some robustness properties of the scheme. The parameter $\delta$ must go to zero in order to enforce a relaxation equilibrium limit characterized by $\pi=p$.

In addition, we propose to introduce a relaxation procedure to approximate the potential $\phi$. As a consequence, $\phi$ is replaced by the new variable $\psi$ satisfying

$$
\partial_{t} \psi=\frac{1}{\delta}(\phi-\psi)
$$


The suggested relaxation model is then given by

$$
\left\{\begin{array}{l}
\partial_{t} \rho+\partial_{x}(\rho u)=0 \\
\partial_{t}(\rho u)+\partial_{x}\left(\rho u^{2}+\pi\right)+\rho \partial_{x} \psi=0, \\
\partial_{t}(\rho E)+\partial_{x}((\rho E+\pi) u)+\rho u \partial_{x} \psi=0, \\
\partial_{t} \pi+u \partial_{x} \pi+\frac{a^{2}}{\rho} \partial_{x} u=\frac{1}{\delta}(p-\pi), \\
\partial_{t} \psi=\frac{1}{\delta}(\phi-\psi) .
\end{array}\right.
$$

Let us emphasize that, in the limit of $\delta=0$, at least formally, we have $\pi=p$ and $\psi=\phi$ and the evolution laws of the density, momentum and energy $(\rho, \rho u$, and $\rho E$, respectively) in (2.6) are thus equivalent to those of (2.1).

For the sake of simplicity in the forthcoming developments, we introduce the condensed form of (2.6), i.e., the compact form of the relaxation system corresponding to (2.2), as

$$
\partial_{t} \mathbf{W}_{\delta}+\partial_{x} \mathbf{F}_{\delta}\left(\mathbf{W}_{\delta}\right)+\mathbf{B}_{\delta}\left(\mathbf{W}_{\delta}\right) \partial_{x} \psi=\frac{1}{\delta} \mathbf{R}_{\delta}\left(\mathbf{W}_{\delta}\right),
$$

which is written in terms of the relaxed variables given by

$$
\begin{aligned}
\mathbf{W}_{\delta} & =(\rho, \rho u, \rho E, \rho \pi, \psi)^{T}, \\
\mathbf{F}_{\delta}\left(\mathbf{W}_{\delta}\right) & =\left(\rho u, \rho u^{2}+\pi,(\rho E+\pi) u,\left(\rho \pi+a^{2}\right) u, 0\right)^{T}, \\
\mathbf{B}_{\delta}\left(\mathbf{W}_{\delta}\right) & =(0, \rho, \rho u, 0,0)^{T}, \\
\mathbf{R}_{\delta}\left(\mathbf{W}_{\delta}\right) & =(0,0,0, \rho(p-\pi), \phi-\psi)^{T} .
\end{aligned}
$$

The set of admissible relaxation state vectors is defined by

$$
\Omega_{\delta}=\left\{\mathbf{W}_{\delta} \in \mathbb{R}^{5} ; \rho>0, u \in \mathbb{R}, \epsilon=E-\frac{u^{2}}{2}>0, \pi \in \mathbb{R}, \psi \in \mathbb{R}\right\},
$$

where the density $\rho$ and specific internal energy $\epsilon$ are positive and must remain positive at all times to be physically meaningful.

\section{The Riemann solution $\mathcal{W}_{\delta}^{\mathcal{R}}$}

We present the solution of the Riemann problem for the relaxation model (2.6) with a vanishing relaxation source term, i.e., $\delta=\infty$. Simultaneously, relevant choices of the parameter $a$ will be specified to enforce a suitable positive preserving property.

First, we consider the algebra of system (2.6). We propose to introduce a change of variables and use the state vector $\mathbf{V}_{\delta}$ defined as

$$
\mathbf{V}_{\delta}=(\rho, u, \epsilon, \pi, \psi)^{T} \text {. }
$$

Then, omitting the source term, (2.6) can be reformulated as

$$
\partial_{t} \mathbf{V}_{\delta}+\mathbf{A}_{\delta}\left(\mathbf{V}_{\delta}\right) \partial_{x} \mathbf{V}_{\delta}=0
$$


where

$$
\mathbf{A}_{\delta}\left(\mathbf{V}_{\delta}\right)=\left(\begin{array}{ccccc}
u & \rho & 0 & 0 & 0 \\
0 & u & 0 & 1 / \rho & 1 \\
0 & \pi / \rho & u & 0 & 0 \\
0 & a^{2} / \rho & 0 & u & 0 \\
0 & 0 & 0 & 0 & 0
\end{array}\right)
$$

An easy computation gives $\mu^{0}=0, \mu_{1}^{u}=\mu_{2}^{u}=u$ and $\mu^{ \pm}=u \pm a / \rho$ as eigenvalues of the above system. It is then clear that the relaxation system (3.1) involves a stationary wave. Following the pioneering work of Greenberg-LeRoux [30,31], we introduce a source term in the associated Riemann solver which naturally comes with a stationary wave (see also [13]). With clear notation, the eigenvectors of $\mathbf{A}_{\delta}\left(\mathbf{V}_{\delta}\right)$ are

$$
\begin{aligned}
& \mathbf{r}^{0}=\left(\rho^{2},-u \rho, \pi, a^{2},\left(u^{2} \rho^{2}-a^{2}\right) / \rho\right)^{T} \\
& \mathbf{r}_{1}^{u}=(1,0,0,0,0)^{T}, \\
& \mathbf{r}_{2}^{u}=(0,0,1,0,0)^{T}, \\
& \mathbf{r}^{ \pm}=\left(\rho^{2}, \pm a, \pi, a^{2}, 0\right)^{T},
\end{aligned}
$$

where $\mathbf{r}_{1}^{u}$ and $\mathbf{r}_{2}^{u}$ are the eigenvectors associated to the double eigenvalue $u$, from now on denoted by $\mu^{u}$. Using simple calculations, we deduce that all the characteristics fields of (2.6) are linearly degenerate. We complete the algebra of system (2.6) by exhibiting the Riemann invariants. Let us recall that the Riemann invariants, denoted $\mathcal{I}$, associated with the eigenvector $\mathbf{r}$, are defined by $\nabla \mathcal{I} \cdot \mathbf{r}=0$. After straightforward computations, with the setting of $\tilde{\pi}=\pi+a^{2} / \rho$ and $\tilde{\epsilon}=\epsilon-\pi^{2} /\left(2 a^{2}\right)$, we find the Riemann Invariants across each wave.

- Across the $\mu^{0}$-wave we have

$$
\mathcal{I}_{1}^{0}=\rho u, \quad \mathcal{I}_{2}^{0}=\tilde{\pi}, \quad \mathcal{I}_{3}^{0}=\tilde{\epsilon}, \quad \mathcal{I}_{4}^{0}=\psi+\frac{u^{2}}{2}-\frac{a^{2}}{2 \rho^{2}}
$$

- Across the $\mu^{u}$-wave we have

$$
\mathcal{I}_{1}^{u}=u, \quad \mathcal{I}_{2}^{u}=\pi, \quad \mathcal{I}_{3}^{u}=\psi
$$

- Across the $\mu^{ \pm}$-waves we have

$$
\mathcal{I}_{1}^{ \pm}=u \pm \frac{a}{\rho}, \quad \mathcal{I}_{2}^{ \pm}=\tilde{\pi}, \quad \mathcal{I}_{3}^{ \pm}=\tilde{\epsilon}, \quad \mathcal{I}_{4}^{ \pm}=\psi
$$

We introduce several notations for the sake of simplicity. We begin by recalling that the specific volume $\tau$ is the reciprocal of the density, namely $\tau=1 / \rho$. Thus, several of 
the quantities previously defined can be written in terms of $\tau$ instead of $\rho$. Additionally, given two constants $X_{L}$ and $X_{R}$, we define

$$
\begin{aligned}
{[X] } & =X_{R}-X_{L} \\
\bar{X} & =\frac{X_{R}+X_{L}}{2} .
\end{aligned}
$$

Now, we fix two admissible states, $\mathbf{W}_{\delta}^{L}$ and $\mathbf{W}_{\delta}^{R}$, in $\Omega_{\delta}$ to define

$$
\mathbf{W}_{\delta}^{0}(x)= \begin{cases}\mathbf{W}_{\delta}^{L} & \text { if } x<0, \\ \mathbf{W}_{\delta}^{R} & \text { if } x>0\end{cases}
$$

the initial data of the Riemann problem for system $(2.6)_{\delta=\infty}$. Thus, we want to find the solution to $\partial_{t} \mathbf{V}_{\delta}+\mathbf{A}_{\delta}\left(\mathbf{V}_{\delta}\right) \partial_{x} \mathbf{V}_{\delta}=0$ with Riemann initial data

$$
\mathbf{V}_{\delta}^{0}= \begin{cases}\mathbf{V}_{L} & \text { if } x<0 \\ \mathbf{V}_{R} & \text { if } x>0\end{cases}
$$

Since all eigenvalues are linearly degenerate, we can compute the exact solution $\mathcal{W}_{\delta}^{\mathcal{R}}$ to the Riemann problem, which has four wave speeds and three intermediate states. To evaluate the intermediate constant states, recall that the Riemann invariants associated with an eigenvector are continuous across the corresponding contact discontinuity. The speeds of the stationary, contact, slowest and fastest waves are defined by $S_{0}, S_{M}, S_{L}$ and $S_{R}$, respectively.

The characterization of the solution is conditioned by the knowledge of the wave ordering, which is not straightforward. By construction, the wave speeds are

$$
S_{0}=0, \quad S_{L}=u_{L}-a \tau_{L}, \quad S_{R}=u_{R}+a \tau_{R} .
$$

Since $\tau_{L}>0$ and $\tau_{R}>0$, we find that the difference $S_{R}-S_{L}=u_{R}-u_{L}+a\left(\tau_{R}+\tau_{L}\right)$ is positive as long as $a$ is large enough. From a numerical point of view, the choice of the parameter $a$ is crucial since it governs (in a way to be defined) the numerical diffusion involved in the scheme. As a consequence, choosing an $a$ too large will produce a very viscous scheme while choosing it too small will lead to an unstable scheme. As usual, the parameter $a$ cannot be smaller than the exact impedance $Z=\rho c$ involved in the initial system $(2.1)$ (see $[7,13,16])$.

Hence, since $S_{R}>S_{L}$ for large enough values of $a$, the wave order problem comes from the position of $S_{R}, S_{L}$ and $S_{M}$ compared to the stationary wave $S_{0}$. In order to cover all possibilities, we set the wave speed

$$
\sigma=\bar{u}-\frac{[\pi]}{2 a}
$$

which corresponds to the velocity in the intermediate region of the Suliciu relaxation model used to approximate the Riemann solution of the Euler equations (see Bouchut 
[13], for instance). Thus, only four distinct cases are possible and they are studied below. In all cases, one can easily recover the intermediate velocity $\sigma$ by setting $[\psi]=0$. Recall that we consider only small values of $[\psi]$, making this a valid approach to segregate Cases 2 and 3.

\subsection{Case 1: $S_{L}>0$}

In this case, we suppose that the wave ordering is $S_{0}=0<S_{L}<S_{M}<S_{R}$. Thus, we search for the exact Riemann solution having the following structure:

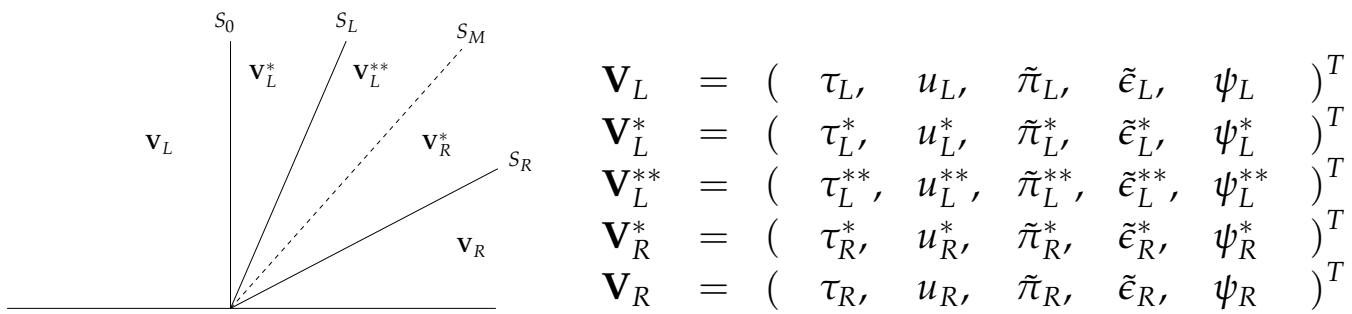

The unknowns that compose this solution are then determined by considering the system given by the continuity of the Riemann invariants (3.2)-(3.4) across each field. Since the velocity and the quantity $\pi$ are constant across $S_{M}$, we have

$$
S_{M}=u_{L}^{* *}=u_{R}^{*}, \quad \pi_{L}^{* *}=\pi_{R^{\prime}}^{*}
$$

respectively. Additionally, we can easily derive the equalities $\psi_{R}=\psi_{R}^{*}=\psi_{L}^{* *}=\psi_{L}^{*}$ and

$$
\begin{aligned}
\tilde{\pi}_{L}=\tilde{\pi}_{L}^{*}=\tilde{\pi}_{L}^{* *}, & \tilde{\pi}_{R}=\tilde{\pi}_{R}^{*}, \\
\tilde{\epsilon}_{L}=\tilde{\epsilon}_{L}^{*}=\tilde{\epsilon}_{L}^{* *}, & \tilde{\epsilon}_{R}=\tilde{\epsilon}_{R}^{*} .
\end{aligned}
$$

Across the $S_{0}$-wave, we have the relations

$$
\begin{aligned}
u_{L} / \tau_{L} & =u_{L}^{*} / \tau_{L}^{*} \\
\psi_{L}+\frac{\left(u_{L}\right)^{2}}{2}-\frac{a^{2}}{2}\left(\tau_{L}\right)^{2} & =\psi_{L}^{*}+\frac{\left(u_{L}^{*}\right)^{2}}{2}-\frac{a^{2}}{2}\left(\tau_{L}^{*}\right)^{2} .
\end{aligned}
$$

Similarly, across the wave with speed $S_{L}$, we have

$$
u_{L}^{*}-a \tau_{L}^{*}=u_{L}^{* *}-a \tau_{L}^{* *},
$$

and, across the $S_{R}$-wave, we get

$$
u_{R}^{*}+a \tau_{R}^{*}=u_{R}+a \tau_{R}
$$

From (3.12a), we obtain

$$
u_{L}^{*}=u_{L} \frac{\tau_{L}^{*}}{\tau_{L}}
$$


We substitute relations (3.15) and $\psi_{L}^{*}=\psi_{R}$ into equation (3.12b) to produce

$$
\left(\tau_{L}^{*}\right)^{2}=\left(\tau_{L}\right)^{2}-\frac{2[\psi]}{\left(u_{L} / \tau_{L}\right)^{2}-a^{2}}
$$

Since the specific volume $\tau_{L}^{*}$ has to be positive, we derive the expression

$$
\tau_{L}^{*}=\tau_{L} \sqrt{1-\frac{2[\psi]}{\left(u_{L}\right)^{2}-a^{2}\left(\tau_{L}\right)^{2}}} .
$$

Recall that $\tilde{\pi}=\pi+a^{2} \tau$. We express the right hand sides of (3.10) in terms of this quantity. Thus, we obtain

$$
\tilde{\pi}_{L}=\pi_{L}^{* *}+a^{2} \tau_{L}^{* *} \quad \text { and } \quad \tilde{\pi}_{R}=\pi_{R}^{*}+a^{2} \tau_{R}^{*}
$$

By performing algebraic manipulations on the above two equations and considering the second equation in (3.9), we derive

$$
\tau_{R}^{*}=\tau_{L}^{* *}+\frac{[\tilde{\pi}]}{a^{2}}
$$

Substitution of $u_{L}^{*}$ from (3.15) into (3.13) gives

$$
u_{L}^{* *}=\left(u_{L} / \tau_{L}-a\right) \tau_{L}^{*}+a \tau_{L}^{* *}=S_{M},
$$

and use of the relation $u_{R}^{*}=u_{R}+a \tau_{R}-a \tau_{R}^{*}=S_{M}$ derived from (3.14) leads to

$$
\tau_{L}^{* *}=\frac{1}{a}\left(u_{R}+a \tau_{R}-a \tau_{R}^{*}-\left(u_{L} / \tau_{L}-a\right) \tau_{L}^{*}\right) .
$$

Then, using (3.17) and (3.19), we deduce the values

$$
\begin{aligned}
\tau_{L}^{* *} & =\frac{1}{2 a}\left(S_{R}-S_{L} \sqrt{1-\frac{2[\psi]}{\left(u_{L}\right)^{2}-a^{2}\left(\tau_{L}\right)^{2}}}-\frac{[\tilde{\pi}]}{a}\right), \\
\tau_{R}^{*} & =\frac{1}{2 a}\left(S_{R}-S_{L} \sqrt{1-\frac{2[\psi]}{\left(u_{L}\right)^{2}-a^{2}\left(\tau_{L}\right)^{2}}}+\frac{[\tilde{\pi}]}{a}\right) .
\end{aligned}
$$

We skip the computation for the other variables since they can be easily deduced from one another. However, from (3.16), we can derive the necessary condition

$$
[\psi]<\frac{1}{2}\left(\left(u_{L}\right)^{2}-a^{2}\left(\tau_{L}\right)^{2}\right),
$$

where $\left(u_{L}\right)^{2}-a^{2}\left(\tau_{L}\right)^{2}>0$ since $S_{L}>0$, that is, $u_{L}+a \tau_{L}>u_{L}-a \tau_{L}>0$. Additionally, in order for $\tau_{L}^{* *}$ and $\tau_{R}^{*}$ to be positive, the following condition must be satisfied:

$$
|[\tilde{\pi}]|<a\left(S_{R}-S_{L} \sqrt{1-\frac{2[\psi]}{\left(u_{L}\right)^{2}-a^{2}\left(\tau_{L}\right)^{2}}}\right) .
$$

If the previous condition is not satisfied, to avoid the appearance of vacuum, we decide to degenerate the $S_{L}$-wave by choosing a larger value of $a$ such that $S_{L}<0<S_{R}, \sigma>0$ and, therefore, switch to Case 2. 
3.2 Case 2: $S_{L}<0<S_{R}, \sigma>0$

Assume that the exact solution follows the wave ordering given by $S_{L}<S_{0}<S_{M}<S_{R}$, which implies looking for the exact Riemann solution of the form:

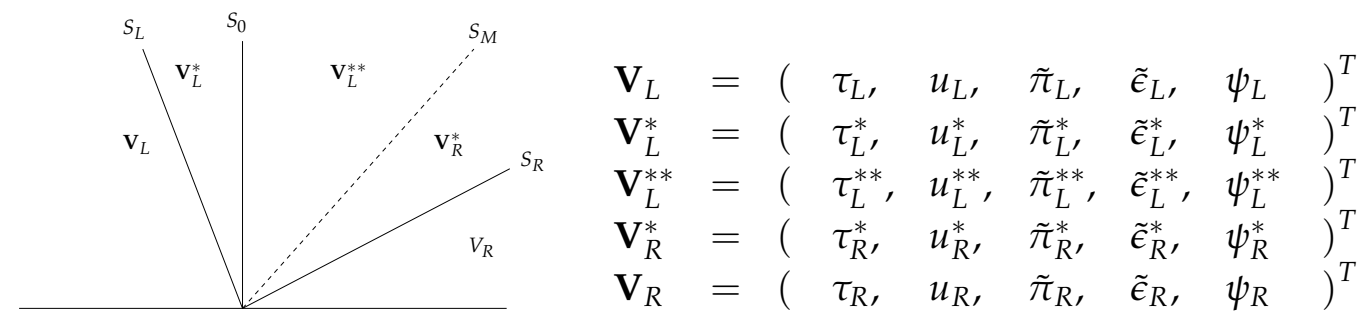

Once again, using the continuity of the Riemann invariants (3.2)-(3.4) across each field, we obtain

$$
\begin{aligned}
u_{L}-a \tau_{L} & =u_{L}^{*}-a \tau_{L}^{*}, \\
u_{L}^{*} / \tau_{L}^{*} & =u_{L}^{* *} / \tau_{L}^{* *}, \\
\psi_{L}^{*}+\frac{\left(u_{L}^{*}\right)^{2}}{2}-\frac{a^{2}}{2}\left(\tau_{L}^{*}\right)^{2} & =\psi_{L}^{* *}+\frac{\left(u_{L}^{* *}\right)^{2}}{2}-\frac{a^{2}}{2}\left(\tau_{L}^{* *}\right)^{2}, \\
u_{R}^{*}+a \tau_{R}^{*} & =u_{R}+a \tau_{R} .
\end{aligned}
$$

Since the velocity and $\pi$ are constant across $S_{M}$, we have

$$
S_{M}=u_{L}^{* *}=u_{R}^{*}, \quad \pi_{L}^{* *}=\pi_{R}^{*}
$$

Additionally, we can easily derive the equalities

$$
\begin{array}{cl}
\psi_{R}=\psi_{R}^{*}=\psi_{L}^{* *}, & \psi_{L}=\psi_{L}^{*} \\
\tilde{\pi}_{L}=\tilde{\pi}_{L}^{*}=\tilde{\pi}_{L}^{* *}, & \tilde{\pi}_{R}=\tilde{\pi}_{R}^{*}, \\
\tilde{\epsilon}_{L}=\tilde{\epsilon}_{L}^{*}=\tilde{\epsilon}_{L}^{* *}, & \tilde{\epsilon}_{R}=\tilde{\epsilon}_{R}^{*} .
\end{array}
$$

By using equation (3.23b), we solve for the velocity $u_{L}^{*}$ as

$$
u_{L}^{*}=u_{L}^{* *} \frac{\tau_{L}^{*}}{\tau_{L}^{* *}}
$$

Direct substitution of relations (3.28), $\psi_{L}^{*}=\psi_{L}$ and $\psi_{L}^{* *}=\psi_{R}$ into equation (3.23c) yields

$$
\left(\frac{\tau_{L}^{*}}{\tau_{L}^{* *}}\right)^{2}=1+\frac{2[\psi]}{\left(u_{L}^{* *}\right)^{2}-a^{2}\left(\tau_{L}^{* *}\right)^{2}} .
$$

From (3.23a) and (3.28), we get

$$
\frac{\tau_{L}^{*}}{\tau_{L}^{* *}}=\frac{u_{L}-a \tau_{L}}{u_{L}^{* *}-a \tau_{L}^{* *}}
$$


By expanding the right hand sides of (3.26), recalling that $\tilde{\pi}=\pi+a^{2} \tau$, we obtain

$$
\tilde{\pi}_{L}=\pi_{L}^{* *}+a^{2} \tau_{L}^{* *} \quad \text { and } \quad \tilde{\pi}_{R}=\pi_{R}^{*}+a^{2} \tau_{R}^{*}
$$

We perform algebraic manipulations on the above two equations and use the second equation in (3.24). Thus, we derive

$$
\tau_{R}^{*}=\tau_{L}^{* *}+\frac{[\tilde{\pi}]}{a^{2}}
$$

which we can substitute into equation (3.23d) to obtain

$$
u_{L}^{* *}=u_{R}^{*}=\alpha-a \tau_{L}^{* *},
$$

with

$$
\alpha=S_{R}-\frac{[\tilde{\pi}]}{a} .
$$

Substitution of relations (3.32) and (3.30) into (3.29) gives the second order polynomial

$$
\left(\alpha-2 a \tau_{L}^{* *}\right)^{2}+\frac{2[\psi]}{\alpha}\left(\alpha-2 a \tau_{L}^{* *}\right)-\left(S_{L}\right)^{2}=0,
$$

which has two real roots. Thus, we find

$$
\tau_{L}^{* *}=\frac{1}{2 a}\left(\alpha+\frac{[\psi]}{\alpha} \pm \sqrt{\left(\frac{[\psi]}{\alpha}\right)^{2}+\left(S_{L}\right)^{2}}\right) .
$$

The suitable root is chosen by considering physical criteria. Recall that the specific volume $\tau_{L}^{* *}$ has to be a positive quantity. Therefore, we select the solution capable of restoring the Riemann solution to the Suliciu model as $[\psi]$ tends to zero. Hence,

$$
\tau_{L}^{* *}=\frac{1}{2 a}\left(\alpha+\frac{[\psi]}{\alpha}+\sqrt{\left(\frac{[\psi]}{\alpha}\right)^{2}+\left(S_{L}\right)^{2}}\right)
$$

and

$$
\tau_{R}^{*}=\frac{1}{2 a}\left(\left(S_{R}+\frac{[\tilde{\pi}]}{a}\right)+\frac{[\psi]}{\alpha}+\sqrt{\left(\frac{[\psi]}{\alpha}\right)^{2}+\left(S_{L}\right)^{2}}\right) .
$$

Direct substitution of $\tau_{L}^{* *}$ into (3.32) leads to

$$
S_{M}=\frac{1}{2}\left(\alpha-\frac{[\psi]}{\alpha}-\sqrt{\left(\frac{[\psi]}{\alpha}\right)^{2}+\left(S_{L}\right)^{2}}\right) .
$$


Now, we verify that if $\alpha$ is large enough such that $|[\psi]|<<|\alpha|$, then the value of the speed $S_{M}$ is approximately

$$
\frac{1}{2}\left(\alpha-\left|S_{L}\right|\right)=\frac{1}{2}\left(u_{L}+u_{R}-\frac{[\pi]}{a}\right)=\sigma .
$$

By performing steps similar to the ones used to find $\tau_{L}^{* *}$, we find the second order polynomial

$$
\left(S_{L}+2 a \tau_{L}^{*}\right)^{2}-\frac{2[\psi]}{S_{L}}\left(S_{L}+2 a \tau_{L}^{*}\right)-\alpha^{2}=0,
$$

which is used to obtain an expression for $\tau_{L}^{*}$. After some algebraic manipulations, we get

$$
\tau_{L}^{*}=\frac{1}{2 a}\left(-S_{L}+\frac{[\psi]}{S_{L}}+\operatorname{sgn}(\alpha) \sqrt{\left(\frac{[\psi]}{S_{L}}\right)^{2}+\alpha^{2}}\right) .
$$

Again, we omit the computation for the remaining unknowns since they can be easily deduced from one another.

\subsection{Case 3: $S_{L}<0<S_{R}, \sigma<0$}

In this case, we assume that the wave ordering is $S_{L}<S_{M}<S_{0}<S_{R}$, and we look for the exact Riemann solution having the structure:

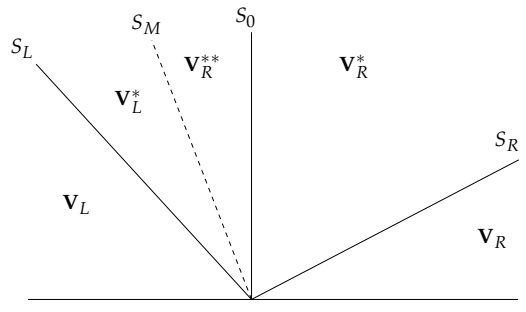

$$
\begin{aligned}
\mathbf{V}_{L} & =\left(\begin{array}{llllll}
\tau_{L}, & u_{L}, & \tilde{\pi}_{L,} & \tilde{\epsilon}_{L}, & \psi_{L}
\end{array}\right)^{T} \\
\mathbf{V}_{L}^{*} & =\left(\begin{array}{llllll}
\tau_{L^{\prime}}^{*} & u_{L^{\prime}}^{*} & \tilde{\pi}_{L^{\prime}}^{*} & \tilde{\epsilon}_{L^{\prime}}^{*} & \psi_{L}^{*}
\end{array}\right)^{T} \\
\mathbf{V}_{R}^{* *} & =\left(\begin{array}{llllll}
\tau_{R}^{* *}, & u_{R}^{* *}, & \tilde{\pi}_{R}^{* *}, & \tilde{\epsilon}_{R}^{* *}, & \psi_{R}^{* *}
\end{array}\right)^{T} \\
\mathbf{V}_{R}^{*} & =\left(\begin{array}{llllll}
\tau_{R}^{*}, & u_{R}^{*}, & \tilde{\pi}_{R}^{*}, & \tilde{\epsilon}_{R}^{*}, & \psi_{R}^{*}
\end{array}\right)^{T} \\
\mathbf{V}_{R} & =\left(\begin{array}{llllll}
\tau_{R}, & u_{R}, & \tilde{\pi}_{R}, & \tilde{\epsilon}_{R}, & \psi_{R}
\end{array}\right)^{T}
\end{aligned}
$$

The continuity of the Riemann invariants (3.2)-(3.4) across the linearly degenerate fields reads

$$
\begin{aligned}
u_{L}-a \tau_{L} & =u_{L}^{*}-a \tau_{L}^{*}, \\
u_{R}^{*} / \tau_{R}^{*} & =u_{R}^{* *} / \tau_{R}^{* *}, \\
\psi_{R}^{*}+\frac{\left(u_{R}^{*}\right)^{2}}{2}-\frac{a^{2}}{2}\left(\tau_{R}^{*}\right)^{2} & =\psi_{R}^{* *}+\frac{\left(u_{R}^{* *}\right)^{2}}{2}-\frac{a^{2}}{2}\left(\tau_{R}^{* *}\right)^{2}, \\
u_{R}^{*}+a \tau_{R}^{*} & =u_{R}+a \tau_{R} .
\end{aligned}
$$

Across the wave with speed $S_{M}$, we have

$$
S_{M}=u_{R}^{* *}=u_{L^{\prime}}^{*} \quad \pi_{R}^{* *}=\pi_{L}^{*} .
$$


In addition, we also derive the equalities

$$
\begin{aligned}
\psi_{L}=\psi_{L}^{*}=\psi_{R}^{* *}, & \psi_{R}=\psi_{R^{\prime}}^{*} \\
\tilde{\pi}_{R}=\tilde{\pi}_{R}^{*}=\tilde{\pi}_{R}^{* *}, & \tilde{\pi}_{L}=\tilde{\pi}_{L^{\prime}}^{*} \\
\tilde{\epsilon}_{R}=\tilde{\epsilon}_{R}^{*}=\tilde{\epsilon}_{R}^{* *}, & \tilde{\epsilon}_{L}=\tilde{\epsilon}_{L}^{*} .
\end{aligned}
$$

In order to find the solution for this case, we use a methodology similar to the one presented in Case 2 and derive the specific volumes

$$
\begin{aligned}
\tau_{R}^{*} & =\frac{1}{2 a}\left(S_{R}+\frac{[\psi]}{S_{R}}-\operatorname{sgn}(\beta) \sqrt{\left(\frac{[\psi]}{S_{R}}\right)^{2}+\beta^{2}}\right), \\
\tau_{R}^{* *} & =\frac{1}{2 a}\left(-\beta+\frac{[\psi]}{\beta}+\sqrt{\left(\frac{[\psi]}{\beta}\right)^{2}+\left(S_{R}\right)^{2}}\right), \\
\tau_{L}^{*} & =\frac{1}{2 a}\left(-\left(S_{L}+\frac{[\tilde{\pi}]}{a}\right)+\frac{[\psi]}{\beta}+\sqrt{\left(\frac{[\psi]}{\beta}\right)^{2}+\left(S_{R}\right)^{2}}\right),
\end{aligned}
$$

with

$$
\beta=S_{L}-\frac{[\tilde{\pi}]}{a} .
$$

We omit the computation for the remaining unknowns, which easily follows from the relations defined in (3.41)-(3.45). However, due to its importance, we give the solution for $S_{M}=u_{R}^{* *}=u_{L}^{*}$, with $u_{L}^{*}=S_{L}+a \tau_{L}^{*}$. Thus,

$$
S_{M}=\frac{1}{2}\left(\beta+\frac{[\psi]}{\beta}+\sqrt{\left(\frac{[\psi]}{\beta}\right)^{2}+\left(S_{R}\right)^{2}}\right) .
$$

If $\beta$ is large enough such that $|[\psi]|<<|\beta|$, then the value of $S_{M}$ is approximately

$$
\frac{1}{2}\left(\beta+\left|S_{R}\right|\right)=\frac{1}{2}\left(u_{L}+u_{R}-\frac{[\pi]}{a}\right)
$$

and consequently, one recovers the speed $\sigma$, as defined in equation (3.8).

\subsection{Case 4: $S_{R}<0$}

Suppose that the exact solution follows the wave ordering $S_{L}<S_{M}<S_{R}<S_{0}=0$ and is composed of five constant states: $\mathbf{V}_{L}, \mathbf{V}_{L}^{*}, \mathbf{V}_{R}^{* *}, \mathbf{V}_{R}^{*}$, and $\mathbf{V}_{R}$. 


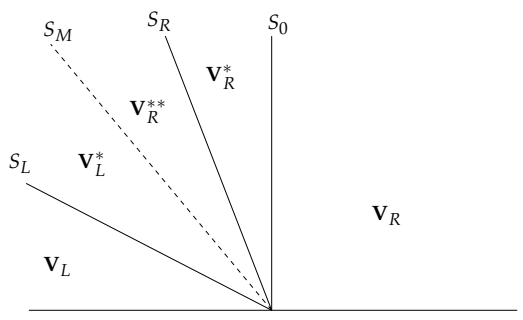

$$
\begin{aligned}
& \mathbf{V}_{L}=\left(\begin{array}{lllll}
\tau_{L}, & u_{L}, & \tilde{\pi}_{L^{\prime}} & \tilde{\epsilon}_{L^{\prime}} & \psi_{L}
\end{array}\right)^{T} \\
& \mathbf{V}_{L}^{*}=\left(\begin{array}{lllll}
\tau_{L^{\prime}}^{*} & u_{L^{\prime}}^{*} & \tilde{\pi}_{L^{\prime}}^{*} & \tilde{\epsilon}_{L^{\prime}}^{*} & \psi_{L}^{*}
\end{array}\right)^{T} \\
& \mathbf{V}_{R}^{* *}=\left(\tau_{R}^{* *}, u_{R}^{* *}, \tilde{\pi}_{R}^{* *}, \tilde{\epsilon}_{R}^{* *}, \psi_{R}^{* *}\right)^{T} \\
& \mathbf{V}_{R}^{*}=\left(\begin{array}{lllll}
\tau_{R^{\prime}}^{*} & u_{R^{\prime}}^{*} & \tilde{\pi}_{R^{\prime}}^{*} & \tilde{\epsilon}_{R^{\prime}}^{*} & \psi_{R}^{*}
\end{array}\right)^{T} \\
& \mathbf{V}_{R}=\left(\begin{array}{lllll}
\tau_{R}, & u_{R}, & \tilde{\pi}_{R}, & \tilde{\epsilon}_{R}, & \psi_{R}
\end{array}\right)^{T}
\end{aligned}
$$

By means of the trivial Riemann invariants, we search for the Riemann solution having the structure presented above. Across $S_{M}$, we have

$$
S_{M}=u_{R}^{* *}=u_{L}^{*}, \quad \pi_{R}^{* *}=\pi_{L}^{*} .
$$

We also derive the equalities $\psi_{L}=\psi_{L}^{*}=\psi_{R}^{* *}=\psi_{R}^{*}$ and

$$
\begin{aligned}
\tilde{\pi}_{R}=\tilde{\pi}_{R}^{*}=\tilde{\pi}_{R}^{* *}, & \tilde{\pi}_{L}=\tilde{\pi}_{L}^{*}, \\
\tilde{\epsilon}_{R}=\tilde{\epsilon}_{R}^{*}=\tilde{\epsilon}_{R}^{* *}, & \tilde{\epsilon}_{L}=\tilde{\epsilon}_{L}^{*} .
\end{aligned}
$$

Additionally, we have the relations

$$
\begin{aligned}
u_{L}-a \tau_{L} & =u_{L}^{*}-a \tau_{L}^{*}, \\
u_{R} / \tau_{R} & =u_{R}^{*} / \tau_{R}^{*} \\
\psi_{R}+\frac{\left(u_{R}\right)^{2}}{2}-\frac{a^{2}}{2}\left(\tau_{R}\right)^{2} & =\psi_{R}^{*}+\frac{\left(u_{R}^{*}\right)^{2}}{2}-\frac{a^{2}}{2}\left(\tau_{R}^{*}\right)^{2}, \\
u_{R}^{*}+a \tau_{R}^{*} & =u_{R}^{* *}+a \tau_{R}^{* *} .
\end{aligned}
$$

Using an approach similar to the one presented in Case 1, we derive the values for the following specific volumes:

$$
\begin{aligned}
\tau_{R}^{*} & =\tau_{R} \sqrt{1+\frac{2[\psi]}{\left(u_{R}\right)^{2}-a^{2}\left(\tau_{R}\right)^{2}}} \\
\tau_{R}^{* *} & =\frac{1}{2 a}\left(-S_{L}+S_{R} \sqrt{1+\frac{2[\psi]}{\left(u_{R}\right)^{2}-a^{2}\left(\tau_{R}\right)^{2}}}+\frac{[\tilde{\pi}]}{a}\right), \\
\tau_{L}^{*} & =\frac{1}{2 a}\left(-S_{L}+S_{R} \sqrt{1+\frac{2[\psi]}{\left(u_{R}\right)^{2}-a^{2}\left(\tau_{R}\right)^{2}}}-\frac{[\tilde{\pi}]}{a}\right) .
\end{aligned}
$$

Again, we decide to skip the computation for the other variables since they can be easily deduced from one another. However, from (3.55), we derive the necessary condition

$$
[\psi]>-\frac{1}{2}\left(\left(u_{R}\right)^{2}-a^{2}\left(\tau_{R}\right)^{2}\right)
$$


In this case, recall that $S_{R}<0$. Therefore, $u_{R}-a \tau_{R}<u_{R}+a \tau_{R}<0$ and the quantity $\left(u_{R}\right)^{2}-a^{2}\left(\tau_{R}\right)^{2}$ is strictly positive. Now, for $\tau_{R}^{* *}$ and $\tau_{L}^{*}$ to be positive specific volumes, we must satisfy the following condition:

$$
|[\tilde{\pi}]|<a\left(-S_{L}+S_{R} \sqrt{1+\frac{2[\psi]}{\left(u_{R}\right)^{2}-a^{2}\left(\tau_{R}\right)^{2}}}\right) .
$$

If the previous condition is not satisfied, in order to avoid the appearance of vacuum, we decide to degenerate the $S_{R}$-wave by choosing a larger value of $a$ such that we can switch to Case 3.

\section{Remarks}

Summarizing the necessary conditions from Cases 2 and 3, we obtain

$$
|[\psi]|<\min (|\alpha|,|\beta|) .
$$

In addition, from Cases 1 and 4, we have

$$
|[\psi]|<\frac{1}{2} \min \left(\left(u_{R}\right)^{2}-a^{2}\left(\tau_{R}\right)^{2},\left(u_{L}\right)^{2}-a^{2}\left(\tau_{L}\right)^{2}\right) .
$$

Thus, we confirm that the solver is robust for small values of $[\psi]$.

\section{1-D relaxation scheme}

Based on the relaxation model (2.6), we present the numerical procedure used to approximate the solution of (2.1). We consider uniform space meshes of size $\Delta x=x_{i+1 / 2}-x_{i-1 / 2}$ for $i \in \mathbb{Z}$. The time increment is given by $\Delta t$ and we note $t^{n+1}=t^{n}+\Delta t$ for $n$ in $\mathrm{N}$.

As mentioned in Section 2, we suppose for the moment that for each time step $t^{n}$, the approximate potential $\phi^{h}$ is known. Hence,

$$
\phi^{h}\left(x, t^{n}\right)=\phi_{i}^{n} \quad \text { if } x \in\left(x_{i-\frac{1}{2}}, x_{i+\frac{1}{2}}\right) .
$$

At time $t=0$, the initial data is given by

$$
\mathbf{W}_{i}^{0}=\frac{1}{\Delta x} \int_{x_{i-\frac{1}{2}}}^{x_{i+\frac{1}{2}}} \mathbf{W}^{0}(x) d x,
$$

where $\mathbf{W}^{0}(x)$ is the initial data function defined in (1.5). Additionally, at time $t^{n}$, we assume that a piecewise constant approximate solution, denoted by $\mathbf{W}^{h}\left(x, t^{n}\right)$, is known and defined by

$$
\mathbf{W}^{h}\left(x, t^{n}\right)=\mathbf{W}_{i}^{n} \quad \text { if } x \in\left(x_{i-\frac{1}{2}}, x_{i+\frac{1}{2}}\right) .
$$




\subsection{Two-step splitting technique}

The obtained approximations are evolved in time using a two-step splitting technique, which will be described in detail subsequently.

\subsubsection{First step: evolution in time}

During the first step, the time evolution is obtained considering the relaxation model (2.6) but omitting the source term, i.e., $\delta=\infty$. More specifically, for all $0<t<\Delta t$, we look for the weak solutions $\mathbf{W}_{\delta}^{h}\left(x, t^{n}+t\right)$ of the Cauchy problem

$$
\partial_{t} \mathbf{W}_{\delta}+\partial_{x} \mathbf{F}_{\delta}\left(\mathbf{W}_{\delta}\right)+\mathbf{B}_{\delta}\left(\mathbf{W}_{\delta}\right) \partial_{x} \psi=0
$$

subject to initial data $\mathbf{W}_{\delta}^{h}\left(x, t^{n}\right)$ given by

$$
\begin{aligned}
\mathbf{W}_{\delta}^{h}\left(x, t^{n}\right) & =\left(\mathbf{W}_{\delta}\right)_{i}^{n} \quad \text { if } x \in\left(x_{i-\frac{1}{2}}, x_{i+\frac{1}{2}}\right) \\
& =\left(\rho_{i}^{n},(\rho u)_{i}^{n},(\rho E)_{i}^{n},(\rho \pi)_{i}^{n}=\rho_{i}^{n} p_{i}^{n}, \psi_{i}^{n}=\phi_{i}^{n}\right) .
\end{aligned}
$$

We note that this initial data coincides with a relaxation equilibrium state since we have enforced $\pi_{i}^{n}=p_{i}^{n}$ and $\psi_{i}^{n}=\phi_{i}^{n}$.

The solution $\mathbf{W}_{\delta}^{h}\left(x, t^{n}+t\right)$ of the above Cauchy problem (4.4)-(4.5) is composed of the juxtaposition of the non-interacting Riemann problem solutions set at each interface $x_{i+1 / 2}$ for $i \in \mathbb{Z}$ and for all $0<t<\Delta t$, with $\Delta t$ small enough such that it satisfies the following CFL like condition:

$$
\frac{\Delta t}{\Delta x} \max _{i \in \mathbb{Z}}\left(\left|\mu_{i+\frac{1}{2}}^{-}\right|,\left|\mu_{i-\frac{1}{2}}^{+}\right|\right) \leq \frac{1}{2},
$$

where the pair $\left(\mu_{i+1 / 2}^{-}, \mu_{i+1 / 2}^{+}\right)$describes the slowest and fastest wave speeds, respectively. The description and details of these waves is given in Section 3.

For the sake of clarity in the notations, we denote by $\mathcal{W}_{\delta}^{\mathcal{R}}\left(\frac{x}{t} ; \mathbf{W}_{\delta}^{L}, \mathbf{W}_{\delta}^{R}\right)$ the Riemann solution associated with (4.4) where the initial data at time $t^{n}$ is given by two constant states $\mathbf{W}_{\delta}^{L}$ and $\mathbf{W}_{\delta}^{R}$ separated by a discontinuity located at $x_{i+\frac{1}{2}}$. Consequently, we have

$$
\mathbf{W}_{\delta}^{h}\left(x, t^{n}+t\right)=\mathcal{W}_{\delta}^{\mathcal{R}}\left(\frac{x-x_{i+\frac{1}{2}}}{t} ;\left(\mathbf{W}_{\delta}\right)_{i}^{n},\left(\mathbf{W}_{\delta}\right)_{i+1}^{n}\right), \quad x \in\left(x_{i}, x_{i+1}\right), \quad 0<t<\Delta t,
$$

where the explicit form of $\mathcal{W}_{\delta}^{\mathcal{R}}$ is described in Section 3. The projection of this solution over the piecewise constant functions reads

$$
\begin{aligned}
\left(\mathbf{W}_{\delta}\right)_{i}^{n+1,-}= & \frac{1}{\Delta x} \int_{x_{i-\frac{1}{2}}}^{x_{i+\frac{1}{2}}} \mathbf{W}_{\delta}^{h}\left(x, t^{n}+\Delta t\right) d x \\
= & \frac{1}{\Delta x} \int_{x_{i-\frac{1}{2}}}^{x_{i}} \mathcal{W}_{\delta}^{\mathcal{R}}\left(\frac{x-x_{i-\frac{1}{2}}}{\Delta t} ;\left(\mathbf{W}_{\delta}\right)_{i-1}^{n},\left(\mathbf{W}_{\delta}\right)_{i}^{n}\right) d x \\
& +\frac{1}{\Delta x} \int_{x_{i}}^{x_{i+\frac{1}{2}}} \mathcal{W}_{\delta}^{\mathcal{R}}\left(\frac{x-x_{i+\frac{1}{2}}}{\Delta t} ;\left(\mathbf{W}_{\delta}\right)_{i}^{n},\left(\mathbf{W}_{\delta}\right)_{i+1}^{n}\right) d x .
\end{aligned}
$$


Let us note that the standard conservative flux balance cannot be reached in the present work because of the potential source term. However, an easy extension of the well-known formalism introduced by Harten, Lax and van Leer [32] gives

$$
\left(\mathbf{W}_{\delta}\right)_{i}^{n+1,-}=\left(\mathbf{W}_{\delta}\right)_{i}^{n}-\frac{\Delta t}{\Delta x}\left(\mathbf{F}_{\delta}^{L}\left(\left(\mathbf{W}_{\delta}\right)_{i}^{n},\left(\mathbf{W}_{\delta}\right)_{i+1}^{n}\right)-\mathbf{F}_{\delta}^{R}\left(\left(\mathbf{W}_{\delta}\right)_{i-1}^{n},\left(\mathbf{W}_{\delta}\right)_{i}^{n}\right)\right),
$$

where

$$
\begin{aligned}
\mathbf{F}_{\delta}^{L}\left(\left(\mathbf{W}_{\delta}\right)_{i}^{n},\left(\mathbf{W}_{\delta}\right)_{i+1}^{n}\right)= & \mathbf{F}_{\delta}\left(\left(\mathbf{W}_{\delta}\right)_{i}^{n}\right) \\
& -\frac{1}{\Delta t} \int_{x_{i}}^{x_{i+\frac{1}{2}}}\left(\mathcal{W}_{\delta}^{\mathcal{R}}\left(\frac{x-x_{i+\frac{1}{2}}}{\Delta t} ;\left(\mathbf{W}_{\delta}\right)_{i}^{n},\left(\mathbf{W}_{\delta}\right)_{i+1}^{n}\right)-\left(\mathbf{W}_{\delta}\right)_{i}^{n}\right) d x, \\
\mathbf{F}_{\delta}^{R}\left(\left(\mathbf{W}_{\delta}\right)_{i}^{n},\left(\mathbf{W}_{\delta}\right)_{i+1}^{n}\right)= & \mathbf{F}_{\delta}\left(\left(\mathbf{W}_{\delta}\right)_{i+1}^{n}\right) \\
& +\frac{1}{\Delta t} \int_{x_{i+\frac{1}{2}}}^{x_{i+1}}\left(\mathcal{W}_{\delta}^{\mathcal{R}}\left(\frac{x-x_{i+\frac{1}{2}}}{\Delta t} ;\left(\mathbf{W}_{\delta}\right)_{i}^{n},\left(\mathbf{W}_{\delta}\right)_{i+1}^{n}\right)-\left(\mathbf{W}_{\delta}\right)_{i+1}^{n}\right) d x .
\end{aligned}
$$

\subsubsection{Second step: relaxation}

During the second step of the scheme, the following system is solved:

$$
\partial_{t} \mathbf{W}_{\delta}=\frac{1}{\delta} \mathbf{R}_{\delta}\left(\mathbf{W}_{\delta}\right)
$$

where the initial data is defined by the piecewise constant approximation $\left(\mathbf{W}_{\delta}\right)_{i}^{n+1,-}$. As $\delta$ tends to zero, the updated approximate equilibrium solution $\mathbf{W}_{i}^{n+1}, \forall i \in \mathbb{Z}$, is given by

$$
\mathbf{W}_{i}^{n+1}=\left(\rho_{i}^{n+1,-},(\rho u)_{i}^{n+1,-},(\rho E)_{i}^{n+1,-}\right)^{T},
$$

and we set $\pi_{i}^{n+1}=p_{i}^{n+1}$ and $\psi_{i}^{n+1}=\phi_{i}^{n+1}$ so that a relaxation equilibrium is reached.

\subsection{Remarks and summary}

The description of the numerical scheme to approximate the solutions to subsystem (2.1) is complete once the relaxation parameter $a$ is correctly characterized. Concerning this parameter, introduced in equation (2.4), let us note that it can be chosen locally at the interfaces since at each interface $x_{i+1 / 2}$, the Riemann solution depends not only on the left and right states $\left(\mathbf{W}_{\delta}\right)_{i}^{n}$ and $\left(\mathbf{W}_{\delta}\right)_{i+1}^{n}$, but also on the parameter $a$. Therefore, this constant $a:=a_{i+1 / 2}$ must be fixed according to the robustness conditions described in Section 3.

Now, involving the usual framework of the finite volume methods, the proposed relaxation scheme is summarized below:

$$
\mathbf{W}_{i}^{n+1}=\mathbf{W}_{i}^{n}-\frac{\Delta t}{\Delta x}\left(\mathbf{F}_{i+\frac{1}{2}}^{L, n}-\mathbf{F}_{i-\frac{1}{2}}^{R, n}\right)
$$


where

$$
\begin{aligned}
& \mathbf{F}_{i+\frac{1}{2}}^{L, n}=\mathbf{F}_{\delta}^{L}\left(\mathbf{W}_{\delta}\left(\mathbf{W}_{i}^{n}\right), \mathbf{W}_{\delta}\left(\mathbf{W}_{i+1}^{n}\right)\right), \\
& \mathbf{F}_{i+\frac{1}{2}}^{R, n}=\mathbf{F}_{\delta}^{R}\left(\mathbf{W}_{\delta}\left(\mathbf{W}_{i}^{n}\right), \mathbf{W}_{\delta}\left(\mathbf{W}_{i+1}^{n}\right)\right),
\end{aligned}
$$

with $\mathbf{W}_{\delta}\left(\mathbf{W}_{i}^{n}\right)=\left(\mathbf{W}_{\delta}\right)_{i}^{n}$ defined according to the relaxation equilibrium, i.e., $\pi_{i}^{n}=p_{i}^{n}$ and $\psi_{i}^{n}=\phi_{i}^{n}$. Indeed, considering the projection step and the relaxation system solution, the numerical fluxes can be written as functions of the relaxation equilibrium states

$$
\begin{aligned}
& \mathbf{F}_{i+\frac{1}{2}}^{L, n}=\mathbf{F}_{i+\frac{1}{2}}^{L, n}\left(\rho_{i}^{n}, \mathbf{u}_{i}^{n},(\rho E)_{i}^{n}, \phi_{i}^{n}, \rho_{i+1}^{n}, \mathbf{u}_{i+1}^{n},(\rho E)_{i+1}^{n}, \phi_{i+1}^{n}\right), \\
& \mathbf{F}_{i+\frac{1}{2}}^{R, n}=\mathbf{F}_{i+\frac{1}{2}}^{R, n}\left(\rho_{i}^{n}, \mathbf{u}_{i}^{n},(\rho E)_{i}^{n}, \phi_{i}^{n}, \rho_{i+1}^{n}, \mathbf{u}_{i+1}^{n},(\rho E)_{i+1}^{n}, \phi_{i+1}^{n}\right) .
\end{aligned}
$$

Let us remark that we kept the notation $\mathbf{F}_{i+1 / 2}^{\alpha, n}$, with $\alpha=L$ or $R$, for the fluctuations since the numerical scheme is not conservative. In fact, the nonconservative operator, coming from $\mathbf{B}(\mathbf{W}) \partial_{x} \phi$ in (2.2), is null except at the interfaces $x_{i+1 / 2}(i \in \mathbb{Z})$ because of the evolution equation (2.5) that governs the relaxation potential. In Section 3, we clearly see that the continuity of the flux function is lost across any interface.

Finally, we proceed to remark that the above presented scheme assumes the approximate potential at time $t^{n}$ to be known, as mentioned at the beginning of this section. This comes from the fact that, as explained in Section 1, we use an operator splitting approach to decompose system (1.2) into two subsystems (1.7) and (1.8). Hence, in the one-dimensional case being considered, once we obtain the approximate solution $\mathbf{W}_{i}^{n+1}$ by means of (4.14), we use $\rho_{i}^{n+1}$ to solve the Poisson equation and thus obtain $\phi_{i}^{n+1}$. We recall that the one-dimensional Poisson equation is

$$
\partial_{x x} \phi=4 \pi G \rho,
$$

and it can be discretized by means of a classical second-order finite difference approach which yields a tridiagonal matrix. There are numerous ways of solving the resulting matrix equation that can be categorized into direct and iterative methods.

\section{$5 \quad$ Numerical results}

The purpose of this section is to illustrate the interest of the proposed relaxation solver for the Euler-Poisson model. In order to address numerical issues, several astrophysical tests are performed. In the first two, we consider a fluid subject to a constant external gravitational field. In the third test, the Lane-Emden equation [36], which describes the hydrostatic equilibrium of a self-gravitating star.

For the problems involving hydrostatic equilibrium flows, the proposed relaxation scheme is compared with a standard fractional step splitting method. This approach also consists on treating subsystems (1.7) and (1.8) individually, with the sole difference 
being that for the former subsystem, one alternates between solving the Euler equations without source terms

$$
\partial_{t} \mathbf{W}+\nabla \cdot \mathbf{F}(\mathbf{W})=0,
$$

and the ordinary differential equation

$$
\partial_{t} \mathbf{W}=-\mathbf{B}(\mathbf{W}) \nabla \phi,
$$

for each time step. Hence, we avoid the necessity of directly incorporating the source terms inside the solver, making this approach quite simple. Hereafter, we will refer to this splitting method as the standard method.

\subsection{1-D Equilibrium flow}

Let us consider a 1-D equilibrium flow. In this case, we neglect the gravitational interactions between particles and suppose that they are subject to a uniform gravitational field. Therefore, we define the gravitational potential $\phi=g x$, with constant $g>0$. We suppose that the fluid is governed by an isentropic equation of state $p=c^{2} \rho$, where $c$ is a non-zero constant. Under this assumption, the hydrostatic equilibrium flow satisfies $\partial_{x} p=-\rho g$ and we deduce the density as a function of position

$$
\rho_{e q}(x)=\rho(0) e^{-g x / c^{2}} .
$$

From this relation, we can define the characteristic scales associated to this experiment: the gravitational length $L_{r e f}=c^{2} / g$ and time $T_{r e f}=\sqrt{L_{r e f} / g}$.

In order to illustrate the accuracy of the proposed method, we propose to compute this equilibrium flow and perform an error analysis in the $L^{2}$ norm. In this context, we set $c=1 \mathrm{~m} . \mathrm{s}^{-1}, L=1 \mathrm{~m}$ and $g=10 \mathrm{~m} \cdot \mathrm{s}^{-2}$. The characteristic scales are then $L_{r e f}=0.1 \mathrm{~m}$ and $T_{\text {ref }} \approx 0.316 \mathrm{~s}$. Hence, we consider meshes with $L \geq L_{r e f}$ and run the experiment a sufficiently long time $T=50 \mathrm{~s}>100 T_{r e f}$. The CFL number is chosen as 0.5. At the initial time, the density is set according to (5.3) with $\rho(0)=10$. Additionally, we have homogeneous Dirichlet boundary conditions for the velocity and non-homogeneous Neumann boundary conditions for the other variables, which are set to ensure the hydrostatic equilibrium.

Figure 1 displays the $L^{2}$ error of the estimated density compared to the analytical solution (5.3) for different mesh sizes $\Delta x$ in a log-log scale. The slope of the error gives the order of accuracy of the method. Using the least squares method, we obtain a linear fit $y=0.9556 x+0.8632$ and thus, the method's order is approximately 0.96 .

On the left in Figure 2, we plot the logarithm of the $L^{2}$ error versus time for 1000 grid points. We can observe that the $L^{2}$ error grows exponentially for a short time and then stabilizes itself, and this holds true for both methods being compared. However, the error for the relaxation method is considerably smaller than the one for the standard method. Additionally, the evolution in time of the $L^{2}$ norm of the velocity, in logarithmic scale, is shown on the right in Figure 2 and one can observe that the velocity field is far lower for the relaxation method. The relaxation scheme clearly enables a better approximation of the equilibrium solution (5.3). 


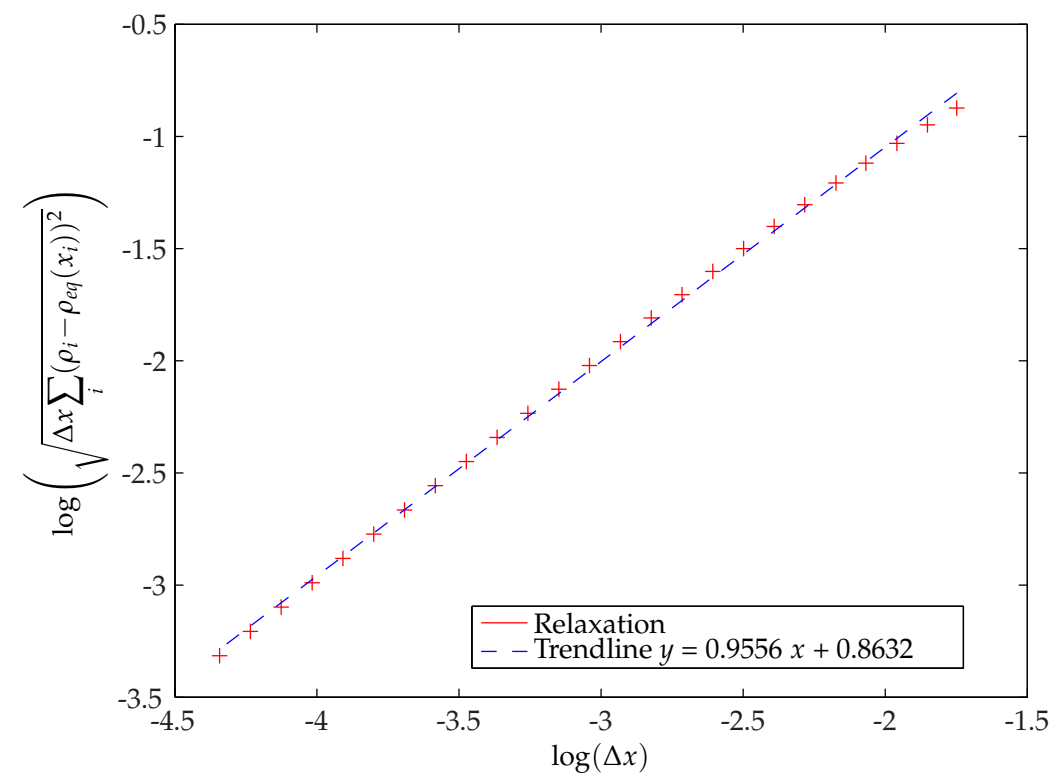

Figure 1: Accuracy of the relaxation method in the case of an equilibrium flow under constant gravitational field and with a fluid governed by an isentropic equation of state.
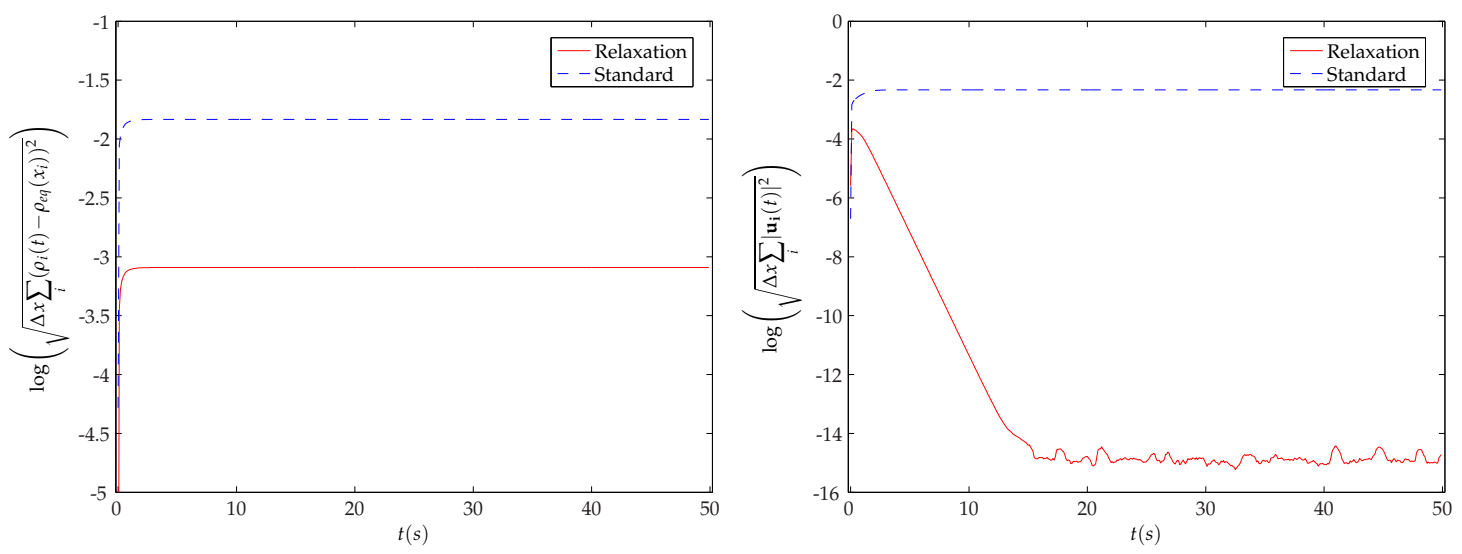

Figure 2: Results in logarithmic scale of the $L^{2}$ error (left) and $L^{2}$ norm of the velocity (right), as functions of time $t$, for Test 5.1 with 1000 grid points. Solid line: relaxation method proposed in this paper; Dashed line: standard method. Since the same half CFL condition (4.7) is used, the total number of time steps for both methods is of the same order of magnitude, specifically, $1.001 \times 10^{5}$ and $1.006 \times 10^{5}$ steps for the relaxation and standard methods, respectively.

\subsection{Rayleigh Taylor instability}

In this section, we propose to validate the proposed method with a Rayleigh Taylor instability occurring as a consequence of a heavy fluid driven into a lighter one under the 
acceleration of a gravitational field. Initially, an unstable interface separates the fluids with different densities.

We carry out this experiment in the two-dimensional plane approximation (translational invariance along the $z$-axis). The derived 1-D numerical method given by (4.14) is easily extended to consider 2-D simulations. As is usual, we suggest

$$
\mathbf{W}_{i j}^{n+1}=\mathbf{W}_{i j}^{n}-\frac{\Delta t}{\Delta x}\left(\mathbf{F}_{i+\frac{1}{2}}^{L, n}-\mathbf{F}_{i-\frac{1}{2}}^{R, n}\right)-\frac{\Delta t}{\Delta y}\left(\mathbf{G}_{j+\frac{1}{2}}^{L, n}-\mathbf{G}_{j-\frac{1}{2}}^{R, n}\right)
$$

where $\mathbf{G}_{j+1 / 2}^{L, n}$ and $\mathbf{G}_{j+1 / 2}^{R, n}$ are the numerical flux functions in the $y$-direction. We emphasize that the 2D Euler-Poisson extension is standard and does not involve difficulties in its derivation.

The two-dimensional computational domain is set to $L_{x}=4 \mathrm{~m}$ and $L_{y}=1 \mathrm{~m}$ and the gravity in the $x$-direction to $g=-1.0 \mathrm{~m} \cdot \mathrm{s}^{-2}$. The domain contains a gas governed by an ideal gas equation of state $p=(\gamma-1) \rho \epsilon$, with $\gamma=1.4$. The gas densities to the right and left of the interface are $\rho_{L}=1 \mathrm{~kg} \cdot \mathrm{m}^{-3}$ and $\rho_{R}=2 \mathrm{~kg} \cdot \mathrm{m}^{-3}$, respectively. The shape of the discontinuity or interface is given by $x=\frac{1}{2} L_{x}\left(1-\frac{1}{10} \cos \left(\left(\frac{y}{L_{y}}-\frac{1}{2}\right) \pi\right)\right)$.

First, this unsteady problem with imposed reflecting boundary conditions is run, using a CFL factor of 0.5 , on a coarse and on a fine grid, composed of $600 \times 150$ and $4000 \times 1000$ points, respectively. The results are displayed in Figure 3. A graphical comparison indicates that a more accurate solution is obtained using the finer grid.
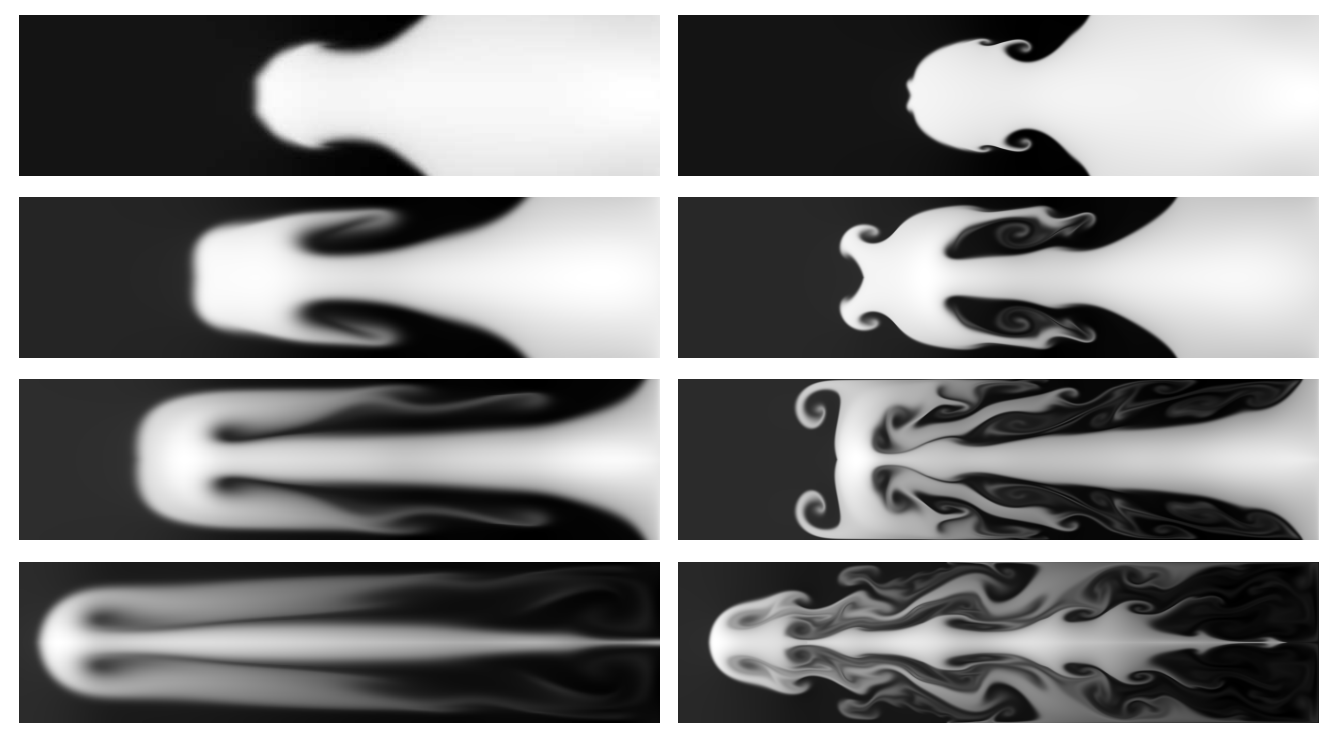

(a) $600 \times 150$ points

(b) $4000 \times 1000$ points

Figure 3: Rayleigh Taylor instability computed with the relaxation scheme proposed in this paper in a 2D computational domain. The results are given at times $t=2.4 s, 4.0 s, 5.6 s, 7.2 s$. 
Additionally, given that approximately first-order scheme is too diffusive, we perform another computation with the relaxation scheme associated with a second-order space method based on a MUSCL reconstruction used in $[47,48]$ associated with four different limiters: minmod [42], van Leer [46], superbee [42], and the positivity preserving limiter $[5,6,45]$. We use the coarse mesh composed of $600 \times 150$ points to show the results in Figure 4. Visually, the positivity preserving limiter is the less diffusive for this particular experiment and clearly more accurate than the first-order scheme on a fine grid.

In all experiments, along the evolution, we obtain the formation of a mushroom cap and the developing of side rolls, as expected. In general, we observe that the Rayleigh Taylor instability has been successfully reproduced.

\subsection{1-D Equilibrium: Lane-Emden equation}

We propose to compute the state of a self gravitational fluid at equilibrium and governed by a polytropic equation of state. In this context, equations (1.1) are rewritten in spherical coordinates $(r, \theta, \varphi)$ and we assume rotational invariance around the axes $e_{\varphi}$ and $e_{\theta}$. The system reduces to

$$
\left\{\begin{array}{l}
\partial_{t}(\rho)+\frac{1}{r^{2}} \partial_{r}\left(r^{2} \rho u_{r}\right)=0, \\
\partial_{t}\left(\rho u_{r}\right)+\frac{1}{r^{2}} \partial_{r}\left(r^{2} \rho u_{r}^{2}\right)+\partial_{r}(p)+\rho \partial_{r}(\phi)=0, \\
\frac{1}{r^{2}} \partial_{r}\left(r^{2} \partial_{r} \phi\right)=4 \pi G \rho .
\end{array}\right.
$$

The polytropic equation of state that governs the gas is given by

$$
p=\kappa \rho^{\gamma}=\kappa \rho^{1+\frac{1}{n}}
$$

where $\kappa$ is the polytropic constant, $\gamma$ the adiabatic exponent and $n$ the polytropic index.

In this context, the 1-D equilibrium flows have been characterized by the Lane-Emden equation [36], which will be derived now. First, assuming the fluid to be at hydrostatic equilibrium ( $\partial_{t}=0$ and $\left.u_{r}=0\right)$, system (5.4) becomes

$$
\left\{\begin{array}{l}
\partial_{r}(p)=-\rho \partial_{r}(\phi), \\
\frac{1}{r^{2}} \partial_{r}\left(r^{2} \partial_{r}(\phi)\right)=4 \pi G \rho .
\end{array}\right.
$$

Combining the momentum equation and the polytropic relation we obtain, after space integration, a relation that links the fluid density to the potential

$$
\rho=\left(\frac{-\phi}{(n+1) \kappa}\right)^{n} \text {. }
$$

Using the Poisson equation, we find the following second order ordinary differential equation for the potential:

$$
\partial_{r}^{2}(\phi)+\frac{2}{r} \partial_{r}(\phi)=4 \pi G\left(\frac{-\phi}{(n+1) \kappa}\right)^{n} .
$$



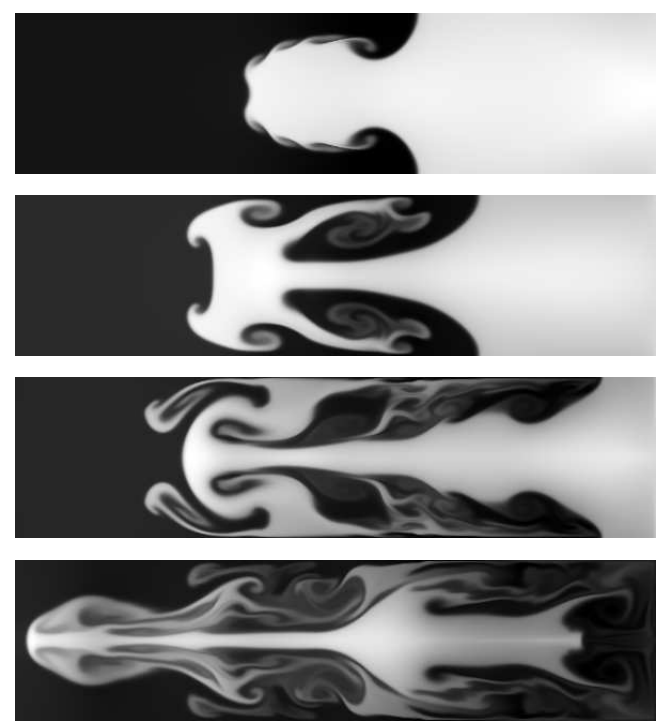

(a) Minmod limiter
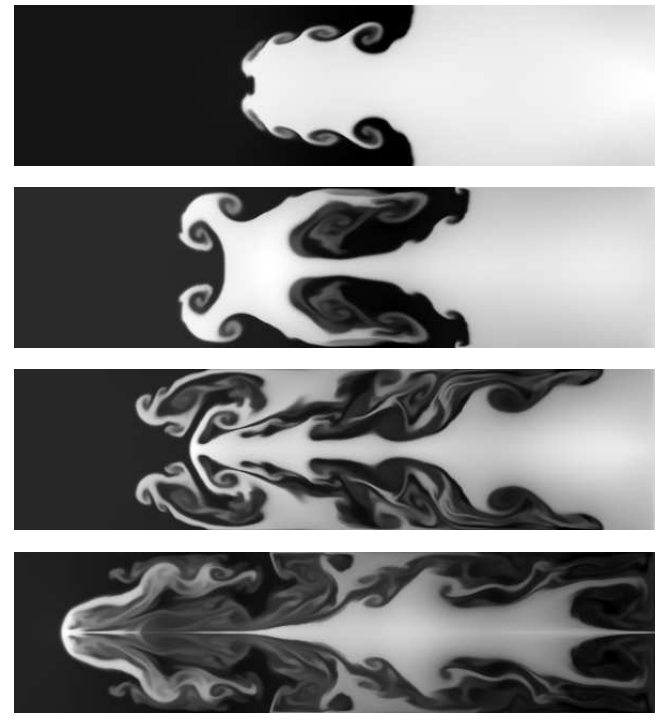

(c) Superbee limiter
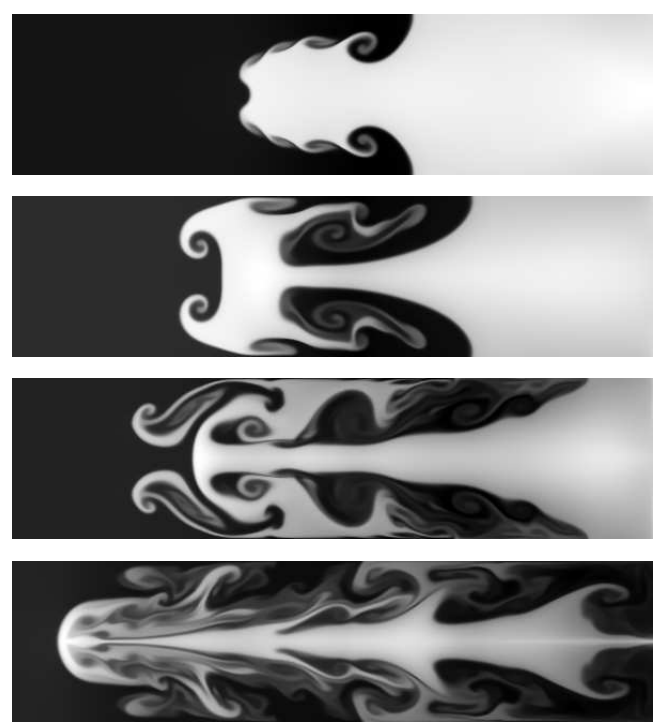

(b) Van Leer limiter
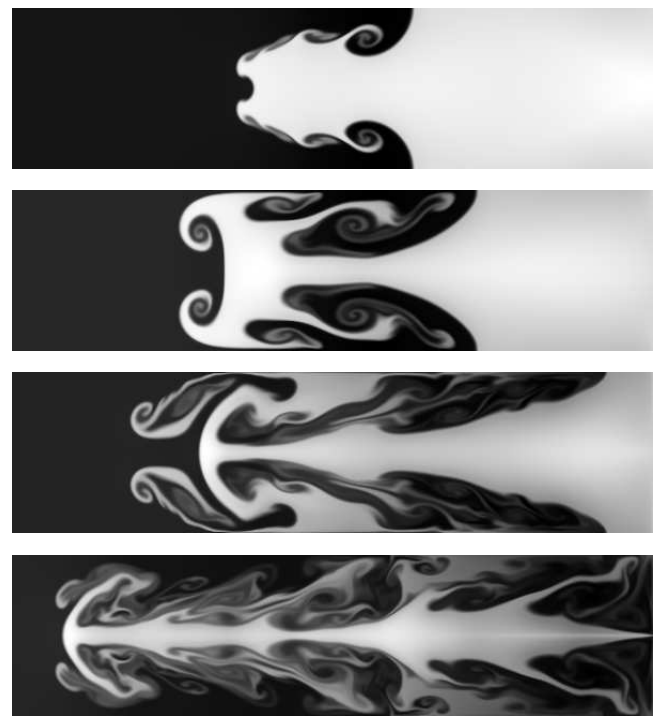

(d) Positivity preserving limiter

Figure 4: Rayleigh Taylor instability computed with a MUSCL-type second-order extension of the relaxation scheme proposed in this paper using different limiters. The computations are performed with $600 \times 150$ points and the results are given at times $t=2.4 s, 4.0 s, 5.6 s, 7.2 s$. 
Defining

$$
\begin{aligned}
& z=A r, \quad w=\frac{\phi}{\phi_{c}}=\left(\frac{\rho}{\rho_{c}}\right)^{\frac{1}{n}}, \\
& A^{2}=\frac{4 \pi G}{(n+1)^{n} \mathcal{K}^{n}}\left(-\phi_{c}\right)^{n-1}=\frac{4 \pi G}{(n+1) \mathcal{K}}\left(\rho_{c}\right)^{\frac{n-1}{n}},
\end{aligned}
$$

with $\phi_{c}$ and $\rho_{c}$ being the potential and the density at the center of the domain, respectively, the differential equation (5.5) becomes the Lane-Emden equation [36]

$$
\frac{1}{z^{2}} \partial_{z}\left(z^{2} \partial_{z} w\right)+w^{n}=0
$$

We are only concerned with solutions to the ordinary differential equation (5.6) that are finite at the center $z=0$. Hence, we assume that $w(z, n)$ is a solution that fulfils the central boundary conditions $w(0, n)=1$ and $\partial_{z} w(0, n)=0$. Additionally, for $n=0,1,5$, we have

$$
w(z, n=0)=1-\frac{z^{2}}{6}, \quad w(z, n=1)=\frac{\sin (z)}{z}, \quad w(z, n=5)=\frac{1}{\sqrt{1+z^{2} / 3}} .
$$

We propose to validate the relaxation method with a polytropic gas of index $n=1$. In order to reproduce this experiment, the numerical codes must be extended to the spherical finite volume formulation. For this, we propose to integrate the system over the spacetime domain $V_{i} \times T^{n}$, where $V_{i}=\left[r_{i-1 / 2}, r_{i+1 / 2}\right] \times[0, \pi] \times[0,2 \pi]$ and $T^{n}=\left[t^{n}, t^{n+1}\right]$. Using the elementary volume expression $d v=r^{2} \sin \theta d r d \theta d \varphi$, we integrate the time differential terms using a finite volume approach and obtain

$$
\begin{aligned}
& \int_{V_{i}} \int_{T_{i}} \partial_{t}(\rho) d t d v=\int_{V_{i}}\left(\rho\left(x, t^{n+1}\right)-\rho\left(x, t^{n}\right)\right) d v=\left|V_{i}\right|\left(\rho_{i}^{n+1}-\rho_{i}^{n}\right), \\
& \int_{V_{i}} \int_{T_{i}} \partial_{t}\left(\rho u_{r}\right) d t d v=\left|V_{i}\right|\left(\left(\rho u_{r}\right)_{i}^{n+1}-\left(\rho u_{r}\right)_{i}^{n}\right),
\end{aligned}
$$

where the volume of a cell is $\left|V_{i}\right|=4 \pi\left(r_{i+1 / 2}^{3}-r_{i-1 / 2}^{3}\right) / 3$. The integration of the divergence terms gives

$$
\begin{aligned}
\int_{T_{i}} \int_{V_{i}} \frac{1}{r^{2}} \partial_{r}\left(r^{2} \rho u_{r}\right) d v d t & =4 \pi \int_{T_{i}} \int_{r_{i-1 / 2}}^{r_{i+1 / 2}} \partial_{r}\left(r^{2} \rho u_{r}\right) d r d t \\
& =4 \pi \Delta t\left(r_{i+1 / 2}^{2}\left(\rho u_{r}\right)_{i+1 / 2}^{L}-r_{i-1 / 2}^{2}\left(\rho u_{r}\right)_{i-1 / 2}^{R}\right),
\end{aligned}
$$

and

$$
\int_{T_{i}} \int_{V_{i}} \frac{1}{r^{2}} \partial_{r}\left(r^{2} \rho u_{r}^{2}\right) d v d t=4 \pi \Delta t\left(r_{i+1 / 2}^{2}\left(\rho u_{r}^{2}\right)_{i+1 / 2}^{L}-r_{i-1 / 2}^{2}\left(\rho u_{r}^{2}\right)_{i-1 / 2}^{R}\right),
$$

with $(\rho u)_{i+1 / 2}^{L, R}$ already defined for the relations (4.10)-(4.11). For the gradient terms, we note that $\rho \partial_{r}(\phi)=0$ over the integration domain $V_{i}$ and we obtain

$$
\int_{T_{i}} \int_{V_{i}} \partial_{r}(p)+\rho \partial_{r}(\phi) d v d t=4 \pi r_{i}^{2} \int_{T_{i}} \int_{r_{i-1 / 2}}^{r_{i+1 / 2}} \partial_{r}(p) d r d t
$$


while

$$
4 \pi r_{i}^{2} \int_{T_{i}} \int_{r_{i-1 / 2}}^{r_{i+1 / 2}} \partial_{r}(p) d r d t=4 \pi r_{i}^{2} \Delta t\left((p)_{i+1 / 2}^{L}-(p)_{i-1 / 2}^{R}\right) .
$$

The numerical scheme associated with system (5.4) and with the relaxation method proposed in this paper is thus given by

$$
\left\{\begin{array}{l}
\frac{\rho_{i}^{n+1}-\rho_{i}^{n}}{\Delta t}+\frac{3 r_{i+1 / 2}^{2}\left(\rho u_{r}\right)_{i+1 / 2}^{L}-3 r_{i-1 / 2}^{2}\left(\rho u_{r}\right)_{i-1 / 2}^{R}}{r_{i+1 / 2}^{3}-r_{i-1 / 2}^{3}}=0, \\
\frac{\left(\rho u_{r}\right)_{i}^{n+1}-\left(\rho u_{r}\right)_{i}^{n}}{\Delta t}+3 \frac{r_{i+1 / 2}^{2}\left(\rho u_{r}^{2}\right)_{i+1 / 2}^{L}-r_{i-1 / 2}^{2}\left(\rho u_{r}^{2}\right)_{i-1 / 2}^{R}+r_{i}^{2}\left((p)_{i+1 / 2}^{L}-(p)_{i-1 / 2}^{R}\right)}{r_{i+1 / 2}^{3}-r_{i-1 / 2}^{3}}=0 .
\end{array}\right.
$$

For the Poisson equation, we propose an implicit scheme deduced from the integration over the volume $V_{i}$ for which we obtain

$$
\frac{3 r_{i+1 / 2}^{2}}{\left(r_{i+1 / 2}^{3}-r_{i-1 / 2}^{3}\right) \Delta r} \phi_{i+1}^{n+1}-\frac{3\left(r_{i+1 / 2}^{2}+r_{i-1 / 2}^{2}\right)}{\left(r_{i+1 / 2}^{3}-r_{i-1 / 2}^{3}\right) \Delta r} \phi_{i}^{n+1}+\frac{3 r_{i-1 / 2}^{2}}{\left(r_{i+1 / 2}^{3}-r_{i-1 / 2}^{3}\right) \Delta r} \phi_{i-1}^{n+1}=4 \pi G \rho_{i}^{n} .
$$

To illustrate the accuracy of the relaxation scheme, we solve the Poisson equation with a standard LU method at each time step since efficiency is not required at the present time.

In order to make numerical comparisons, the standard method is introduced. The first step is devoted to the resolution of system (5.4) with $\partial_{r}(\phi)=0$ by using the proposed relaxation scheme in the spherical formulation (5.7). In the second step, we solve the following system:

$$
\left\{\begin{array}{l}
\partial_{t}(\rho)=0 \\
\partial_{t}\left(\rho u_{r}\right)+\rho \partial_{r}(\phi)=0 \\
\frac{1}{r^{2}} \partial_{r}\left(r^{2} \partial_{r} \phi\right)=4 \pi G \rho .
\end{array}\right.
$$

As a numerical test, we consider a polytropic gas governed by $p=\kappa \rho^{1+1 / n}$ with the polytropic constant $\kappa=1000$ and index $n=1$. The size of the experimental domain is $\left[0, L_{r}\right]$ with $L_{r}=6.0 \times 10^{5} \mathrm{~m}$. At the center of the domain, we impose $\rho_{c}=10 \mathrm{~kg} \cdot \mathrm{m}^{-3}$. We set the number of points to 100 and the final time of the experiment to $1.0 \times 10^{7} \mathrm{~s}$. The density approximation is displayed in Figure 5.

Additionally, we observe the parasitic currents evolution during the calculation. For this, we plot the evolution in time of the $L^{2}$ norm of the velocity, in logarithmic scale, (Figure 6). The velocity field is far lower for the relaxation method than for the standard method, i.e., for the relaxation scheme, we have an approximate residual velocity of 0.01 and for the standard scheme, we get approximately 0.3 . The relaxation scheme enables a better approximation of equilibrium solutions because it generates very low parasitic currents. 


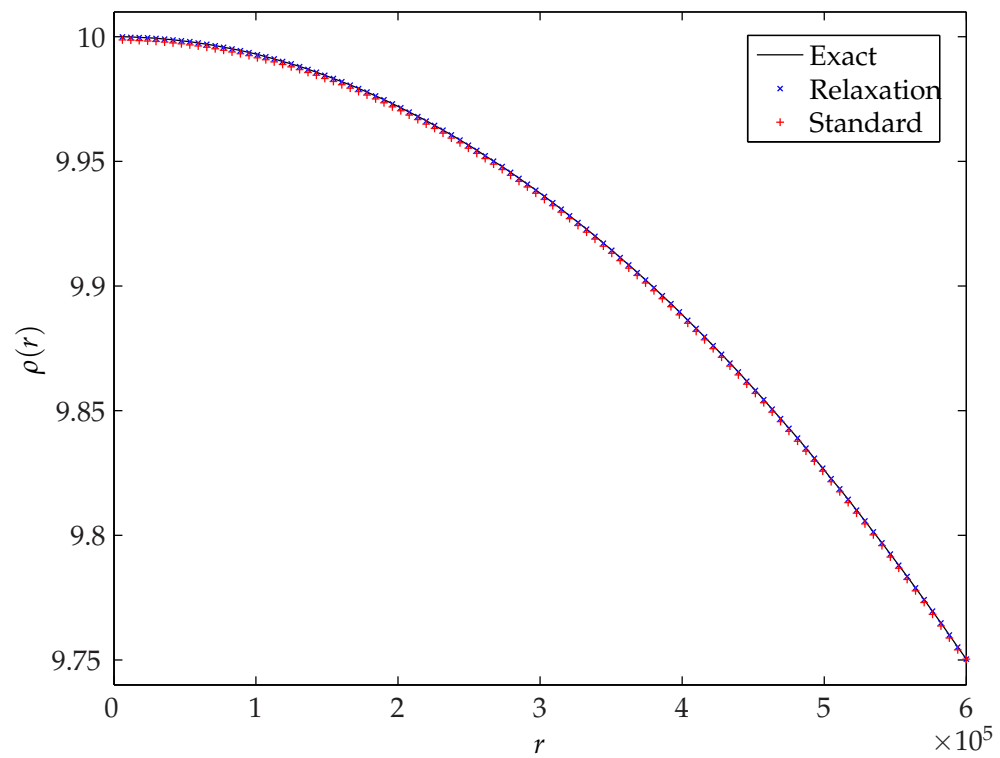

Figure 5: Numerical densities for Test 5.3 obtained by the relaxation and standard methods, compared to the exact solution $\rho(r)=10 * \sin (z) /(z)$ with $z=A r$. The total number of time steps required for this simulation is approximately $1.065 \times 10^{7}$ steps for both methods.

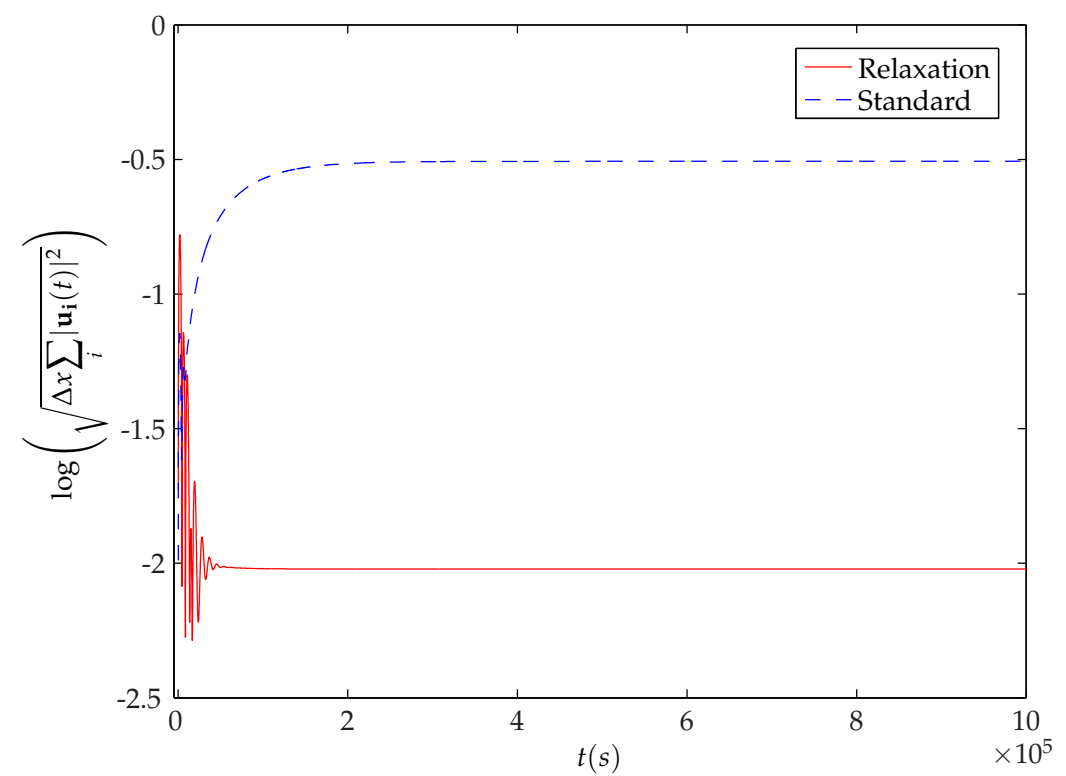

Figure 6: $L^{2}$ norm of the velocity for Test 5.3, in logarithmic scale, as a function of time $t$, with 100 grid points. Solid line: relaxation method proposed in this paper; Dashed line: standard method. 


\section{Conclusions}

In this paper, we have derived a Suliciu-type relaxation solver to approximate the solutions to the Euler-Poisson system. In view of astrophysical simulations, the asymptotic regime of self gravitational equilibrium flows must be preserved. Although several models have been developed for this specific problem [21,22], they become far too complex in 3-D or when an implicit formulation is required. We have presented here a simpler numerical scheme based on a Godunov-type solver deduced from a relaxation system to resolve accurately steady equilibrium flows. We proved the accuracy of our relaxation method on an equilibrium flow under constant gravitational forces. Then, we proposed the numerical simulation of a Rayleigh Taylor instability, an unsteady problem, which was successfully reproduced by the proposed numerical scheme. Finally, we reconsidered our scheme in spherical coordinates in order to recover precisely the solution of the Lane-Emden equation. Future work will be devoted to a well-balanced time implicit formulation of the method and simulations of 3-D astrophysical problems.

\section{Acknowledgments}

This research was partially supported by the A.N.R. (Agence Nationale de la Recherche) through the projects SiNeRGHY (ANR-06-CIS6-009-01) and Anemos (ANR-11-MONU002). The authors would also like to thank Neil Vaytet for his careful reading of the manuscript.

\section{References}

[1] R. Abgrall, S. Karni, A relaxation scheme for the two-layer shallow water system, Hyperbolic Problems: Theory, Numerics, Application, S. Benzoni-Gavage and D. Serre Editors, Springer, 135-144, 2008.

[2] D. Aregba-Driollet, C. Berthon, Numerical approximation of Kerr-Debye equations, preprint hal00293728.

[3] S. Balbus, J.C.B. Papaloizou, On the dynamical foundations of $\alpha$ disks, Astrophysical Journal 521 (1999), p. 650.

[4] M. Baudin, C. Berthon, F. Cocquel, R. Masson, Q. H. Tran, A relaxation method for two-phase flow models with hydrodynamic closure law, Numer. Math. 99 (2005), 411-440.

[5] C. Berthon, Stability of the MUSCL schemes for the Euler equations, Comm. Meth. Sci. 3 (2005), 133-158.

[6] C. Berthon, Robustness of MUSCL schemes for 2D unstructured meshes, J. Comput. Phys. 218 (2006), 495-509.

[7] C. Berthon, Numerical approximations of the 10-moment Gaussian closure, Math. Comput. 75 (2006), 1809-1831.

[8] C. Berthon, B. Braconnier, B. Nkonga, Numerical approximation of a degenerated nonconservative multifluid model: relaxation scheme, International Journal for Numerical methods in Fluids 48 (2005), 85-90. 
[9] C. Berthon, M. Breuss, M. O. Titeux, A relaxation scheme for pressureless gas dynamics, Num. Method Partial Diff. Equations 22 (2006), 484-505.

[10] C. Berthon, P. Charrier, B. Dubroca, An HLLC scheme to solve the M1 Model of radiative transfer in two space dimensions, J. Sci. Comput. 31 (2007), 347-389.

[11] C. Berthon, F. Marche, A positive preserving hight order VFRoe scheme for shallow water equations: A class of relaxation schemes, SIAM J. Sci. Comput. 30 (2008), 2587-2612.

[12] F. Bouchut, Entropy satisfying flux vector splittings and kinetic BGK models, Numer. Math. 94 (2003), 623-672.

[13] F. Bouchut, Nonlinear Stability of Finite Volume Methods for Hyperbolic Conservation Laws, and Well-Balanced Schemes for Sources, Frontiers in Mathematics, Birkhauser, 2004.

[14] F. Bouchut, T. Morales, An entropy satisfying scheme for two-layer shallow water equations with uncoupled treatment, M2AN 42 (2008), 683-698.

[15] C. Chalons, F. Coquel, Navier-Stokes equations with several independent pressure laws and explicit predictor-corrector schemes, Numer. Math. 101 (2005), 451-478.

[16] C. Chalons, J.-F. Coulombel, Relaxation approximation of the Euler equations, J. Math. Anal. Appl. 348 (2008), 872-893.

[17] G. Q. Chen, C. D. Levermore, T. P. Liu, Hyperbolic conservation laws with stiff relaxations terms and entropy, Comm. Pure Appl. Math. 47 (1995), 787-830.

[18] J.-P. Chièze, Elements of Hydrodynamics Applied to the Interstellar Medium, Starbursts: Triggers, Nature, and Evolution, Les Houches School, September 17-27, 1996. ESP Sciences 1998, p. 77, Editors Bruno Guiderdoni and Ajit Kembhavi.

[19] F. Coquel, B. Perthame, Relaxation of energy and approximate Riemann solvers for general pressure laws in fluid dynamics, SIAM J. Numer. Anal 35 (1998), 2223-2249.

[20] P. Crispel, P. Degond, M.-H. Vignal, An asymptotically stable discretization for the Euler-Poisson system in the quasineutral limit, C. R. Acad. Sci. Paris Ser. I (2005), 323-328.

[21] P. Crispel, P. Degond, M.-H. Vignal, An asymptotic preserving scheme for the two-fluid EulerPoisson model in the quasineutral limit, J. Comput. Phys. 223 (2007), 208-234.

[22] P. Degond, F. Deluzet, A. Sangam, M.-H. Vignal, An Asymptotic Preserving scheme for the Euler equations in a strong magnetic field, J. Comput. Phys. 228 (2009), 3540-3558.

[23] P. Degond, S. Jin, J.-G. Liu, Mach-number uniform asymptotic-preserving Gauge schemes for compressible flows, Bull. Inst. Math., Acad. Sinica (New Series) 2 (2007), 851-892.

[24] P. Degond, J.-G. Liu, M.-H. Vignal, Analysis of an asymptotic preserving scheme for the EulerPoisson system in the quasineutral limit, SIAM J. Numer. Anal. 46 (2008), 1298-1322.

[25] S. Fabre, Stability analysis of the Euler-Poisson equations, J. Comput. Phys. 101 (1992), 445-451.

[26] T. Gallouet, J. M. Hérard, N.Seguin, Some approximate Godunov schemes to compute shallowwater equations with topography, Computers and Fluids 32 (2003), 479-513.

[27] J. M. Gallardo, C. Parés, M. Castro, On a well-balanced high-order finite volume scheme for shallow water equations with topography and dry areas, J. Comput. Phys. 227 (2007), 574-601.

[28] E. Godlewski, P.A. Raviart, Hyperbolic systems of conservations laws, Applied Mathematical Sciences, 118, Springer, 1996.

[29] M. Gonzáles, E. Audit, P. Huynh, HERACLES: a three-dimensional radiation hydrodynamics code, Astronomy Astrophysics 464 (2007), 429-435.

[30] J. M. Greenberg, A. Y. Leroux, A well-balanced scheme for the numerical processing of source terms in hyperbolic equations, SIAM J. Numer. Anal. 33 (1996), 1-16.

[31] J. M. Greenberg, A. Y. Leroux, R. Baraille, A. Noussair, Analysis and approximation of conservation laws with source terms, SIAM J. Numer. Anal. 34 (1997), 1980-2007.

[32] A. Harten, P.D. Lax, B. Van Leer, On upstream differencing and Godunov-type schemes for hyper- 
bolic conservation laws, SIAM 25 (1983), 33-61.

[33] R. A. James, The solution of Poisson's equation for isolated source distributions, J. Comput. Phys. 25 (1977), 71-93.

[34] S. Jin, A steady-state capturing method for hyperbolic systems with geometrical source terms, M2AN Math. Model. Numer. Anal. 35 (2001), 631-645.

[35] S. Jin, Z. P. Xin, The relaxation schemes for systems of conservation laws in arbitrary space dimensions, Comm. Pure Appl. Math. 48 (1995), 235-278.

[36] R. Kippenhahn, A. Weigert, Stellar Structure and Evolution, Springer, Berlin (a.o.), 1990.

[37] R. J. LeVeque, Balancing source terms and flux gradients in high-resolution Godunov methods: the quasi-steady wave-propagation algorithm, J. Comput. Phys. 146 (1998), 346-365.

[38] R. J. LeVeque, M. Pelanti, A class of approximate Riemann solvers and their relation to relaxation schemes, J. Comput. Phys. 172 (2001), 572-591.

[39] Y. Li, Convergence of the nonisentropic Euler-Poisson equations to incompressible type Euler equations, J. Math. Anal. Appl. 342 (2008), 1107-1125.

[40] R. Natalini, F. Rousset, Convergence of a singular Euler-Poisson approximation of the incompressible Navier-Stokes equations, Proc. Amer. Math. Soc. 134 (2006), 2251-2258.

[41] S. Noelle, N. Pankratz, G. Puppo, J. R. Natvig, Well-balanced finite volume schemes of arbitrary order of accuracy for shallow water flows, J. Comput. Phys. 213 (2006), 474-499.

[42] P.L. Roe, Characteristic-based schemes for the Euler equations, Ann. Rev. Fluid Mech. 18 (1986), 337-365.

[43] I. Suliciu, On modelling phase transitions by means of rate-type constitutive equations, shock wave structure, Int. J. Engrg. Sci. 28 (1990), 829-841.

[44] I. Suliciu, Some stability-instability problems in phase transitions modelled by piecewise linear elastic or viscoelastic constitutive equations, Int. J. Engrg. Sci. 30 (1992), 483-494.

[45] A. Suresh, Positivity-preserving schemes in multidimensions, SIAM Sci. Comput. 22 (2000), 1184-1198.

[46] B. van Leer, Towards the ultimate conservative difference scheme II. Monotonicity and conservation combined in a second order scheme, J. Comput. Phys. 14 (1974), 361-70.

[47] B. van Leer, Towards the ultimate conservative difference scheme V. A second-order sequel to Godunov's method, J. Comput. Phys. 32 (1979), 101-136.

[48] B. van Leer, On the relation between the upwind-differencing schemes of Godunov, Engquist-Osher and Roe, SIAM J. Sci. Statist. Comput. 5 (1984), 1-20.

[49] H. Weyl, Shock waves in arbitrary fluids, Comm. Pure Appl. Math. 2 (1949), 103-122.

[50] J. Whitham, Linear and Nonlinear Waves, Wiley, New-York, 1974. 